\title{
Multiple Multidimensional Morse Wavelets
}

\author{
Georgios Metikas and Sofia C. Olhede
}

\begin{abstract}
This paper defines a set of operators that localize a radial image in space and radial frequency simultaneously. The eigenfunctions of the operator are determined and a nonseparable orthogonal set of radial wavelet functions are found. The eigenfunctions are optimally concentrated over a given region of radial space and scale space, defined via a triplet of parameters. Analytic forms for the energy concentration of the functions over the region are given. The radial function localization operator can be generalised to an operator localizing any $L^{2}\left(\mathbb{R}^{2}\right)$ function. It is demonstrated that the latter operator, given an appropriate choice of localization region, approximately has the same radial eigenfunctions as the radial operator. Based on a given radial wavelet function a quaternionic wavelet is defined that can extract the local orientation of discontinuous signals as well as amplitude, orientation and phase structure of locally oscillatory signals. The full set of quaternionic wavelet functions are component by component orthogonal; their statistical properties are tractable, and forms for the variability of the estimators of the local phase and orientation are given, as well as the local energy of the image. By averaging estimators across wavelets, a substantial reduction in the variance is achieved.
\end{abstract}

Index Terms - Analytic signal, image analysis, monogenic signal, Riesz transform, scalogram, wavelets.

\section{INTRODUCTION}

$\mathbf{L}$ OCALIZED analyses in one-dimension(al) (1-D) have proven to be remarkably successful-notably so wavelet analyses. The latter is based on the idea that observed signals varying over an increasing argument, time say, exhibit disparate and highly localized behavior associated with variations at a particular scale and at particular time points. Analysis is based on the wavelet transform (WT), given for signal $g(t)$ using mother wavelet $\psi(t)$ via

$$
\begin{aligned}
w_{\psi}(a, b ; g) & =\left\langle\psi_{a, b}, g\right\rangle \\
& =\int_{-\infty}^{\infty} g(t)|a|^{-1 / 2} \psi^{*}\left(\frac{t-b}{a}\right) d t
\end{aligned}
$$

where $a$ is referred to as the scale, $b$ the translation and $*$ denotes conjugation. Equation (1) facilitates the recognition of patterns specific to time points $b$ and length scales associated with scale $a$, if the function $\psi(\cdot)$ is chosen such that the support of $\psi(\cdot)$ is essentially limited to a region near the origin, and the support of the Fourier transform of $\psi(\cdot)$, is essentially limited to a region near some reference frequency $f_{\max } \neq 0$. A function cannot have compact support in the two domains simultaneously and to

Manuscript received September 8, 2005; revised May 14, 2006. The work of G. Metikas was supported by an EPSRC Grant. The associate editor coordinating the review of this paper and approving it for publication was Dr. Antonia Papandreou-Suppappola.

The authors are with the Department of Mathematics, Imperial College London, SW7 2AZ, London, U.K. (e-mail: s.olhede@imperial.ac.uk).

Digital Object Identifier 10.1109/TSP.2006.887562 measure the localization of an arbitrary function $\psi(x)$, localization operators were introduced. The operators generalize truncation operators, that act consecutively in time and frequency [1], to operators simultaneously localizing a signal in time and frequency (or equivalently scale) over a given region [2], [3]. The eigenfunctions of such operators are optimally localized with respect to a given time-frequency domain and the problem of defining appropriate operators in one dimension and calculating their eigenfunctions has been considered in detail [2]-[5].

Extending 1-D local decomposition methods to 2-D analysis is nontrivial as variation in the spatial variable is often associated with a specific direction, that in general, may not be aligned with the observational axes. If a local oscillation at angle $\nu(\boldsymbol{x})$ with respect to the axes and frequency $f_{1}(\boldsymbol{x})$ is considered, then a tensor product local decomposition would represent the structure as oscillations in both axes with local frequency $f_{1}(\boldsymbol{x}) \cos (\nu(\boldsymbol{x}))$ and $f_{1}(\boldsymbol{x}) \sin (\nu(\boldsymbol{x}))$. A local decomposition in the correct direction would yield constant behavior in one orthogonal axes and an oscillation with frequency $f_{1}(\boldsymbol{x})$ in the other. This gives a simpler representation. Naturally either of the two representations describes the same structure in two different ways, where a directional representation has the potential of additional compression and elegance. The latter features provide arguments in favour of using a decomposition that can be tuned to a given direction. In 2-D, localization will be made to spatial point $\boldsymbol{b}=\left[b_{1}, b_{2}\right]^{T}$, in scale to $a$ and in orientation to angle $\theta \in[0,2 \pi)$, following work by Antoine, Murenzi $\&$ Vandergheynst [6]. For a discussion of recently developed multidimensional decompositions, see Antoine, Murenzi, Vandergheynst, and Ali [7]. To construct a 2-D continuous wavelet decomposition we define the dilation $\left(\mathcal{D}_{a}\right)$, and translation $\left(\mathcal{T}_{\boldsymbol{b}}\right)$ operators for $g(\boldsymbol{x}) \in L^{2}\left(\mathbb{R}^{d}\right), d=1,2, \ldots$ with $\boldsymbol{x}, \boldsymbol{b} \in \mathbb{R}^{d}$, by $\mathcal{D}_{a} g(\boldsymbol{x})=|a|^{-d / 2} g\left(a^{-1} \boldsymbol{x}\right)$ and $\mathcal{T}_{\boldsymbol{b}} g(\boldsymbol{x})=g(\boldsymbol{x}-\boldsymbol{b})$, and define $\boldsymbol{\xi}=[a, \theta, \boldsymbol{b}]^{T}$. The WT is constructed from the family of functions $\left\{\psi_{\boldsymbol{\xi}}(\cdot)\right\}$ defined in terms of the mother wavelet function $\psi(\boldsymbol{x})$ by

$$
\begin{aligned}
\psi_{\boldsymbol{\xi}}(\boldsymbol{x}) & =\psi_{a, \theta, \boldsymbol{b}}(\boldsymbol{x})=\mathcal{R}_{\theta} \mathcal{D}_{a} \mathcal{T}_{\boldsymbol{b}} \psi(\boldsymbol{x}) \\
& =|a|^{-1} \psi\left(\boldsymbol{r}_{-\theta} a^{-1}(\boldsymbol{x}-\boldsymbol{b})\right)
\end{aligned}
$$

with $\boldsymbol{r}_{\theta}$ given as the rotation matrix [7]. The 2-D continuous wavelet decomposition of image $g(\boldsymbol{x})$ is constructed from

$$
\begin{aligned}
w_{\psi}(a, \theta, \boldsymbol{b} ; g) & =\left\langle\psi_{a, \theta, \boldsymbol{b}}, g\right\rangle \\
& =\iint_{\boldsymbol{R}^{2}} g(\boldsymbol{x}) \psi_{a, \theta, \boldsymbol{b}}^{*}(\boldsymbol{x}) d^{2} \boldsymbol{x} .
\end{aligned}
$$

Equation (3) is a continuous space decomposition of an observed signal, and will be used for analysis of observed signals, rather than serve as the basis of an image estimation procedure, which requires perfect reconstruction decomposition filters. The 
decomposition of (3) will, with an appropriate choice of wavelet function, uncover/disentangle behavior across specific spatial points, scales and orientation, and facilitate the analysis of nonstationary image phenomena. In perfect analog with 1-D theory an appropriate choice of wavelet function will correspond to using a mother wavelet function that is local to, or essentially supported near, $\boldsymbol{x}=\mathbf{0}$ and some particular choice of scale and direction localization. Existing continuous wavelet methods that deal with the orientation of the image explicitly are based on directionally selective filters, or directional wavelets.

Antoine, Murenzi, and Vandergheynst [6] defined complex directional wavelets with a preferred orientation in the frequency domain, as their frequency support is limited to a predefined cone, parameterized via the opening and closing angles of the cone [6, pp. 324, 325]. Highly directional wavelets, by necessity, filter the image nonisotropically. For images containing directional structures this will lead to very useful representations of these directional structures. We wish to construct wavelets where the local decomposition coefficients are calculated by averaging the image over a region in the spatial domain which is defined in terms of radial distances from its centre point, and associates oscillations with approximately the same period. This choice associates energy at spatial point $\boldsymbol{x}$ and spatial frequency $\boldsymbol{f}$ with energy at $\boldsymbol{x}^{\prime}$ and $\boldsymbol{f}^{\prime}$ depending on the value of $\left|\boldsymbol{x}-\boldsymbol{x}^{\prime}\right|$ and $|\boldsymbol{f}|-\left|\boldsymbol{f}^{\prime}\right|$.

As aforementioned, an isotropic WT is used to separate out disparate components occurring at different scales and/or at different spatial locations. The directional structure of the image will at this stage not be considered explicitly. To facilitate the radial separation of structures, wavelets that are optimally concentrated in radial space and radial frequency are required. We define a family of radial 2-D localization operators and find the radial eigenfunctions of any given operator in this family, denoted the isotropic Morse wavelets. The eigenfunctions can be considered as optimally concentrated over a given domain. Any operator in this family of localization operators is characterized via three parameters: the two shape parameters that determine the localization region, and thus the compromise between spatial position and spatial frequency localization, and the hypervolume parameter. Any choice of the parameters fixes a particular operator that in turn possesses a family of eigenfunctions. These functions are orthogonal, and indexed via an integer value $n$, but do not depend on the value of the hypervolume. The eigenvalues explicitly give the radial concentration of the eigenfunctions. The eigenfunctions are related, but not equivalent, to the even eigenfunctions of the 1-D Morse localization operator [3].

The second part of the paper discusses the choice of local directional representation. The directional representation will correspond to the extension of the isotropic wavelets into a direction sensitive analysis tool representing the local oscillatory structure in terms of a phase, similar in nature to the 1-D analysis of [8]. For every fixed value of $n$, and given radial eigenfunction wavelet, an additional pair of functions are defined for each Morse wavelet, constructed in analog to analytic 1-D wavelets. In 1-D, an even wavelet function is complemented by its Hilbert transform and the two are combined into an analytic wavelet. Analytic wavelets are used to represent local phase structure. In
2-D, several additional wavelets are calculated at each spatial point. Each additional set of wavelets is considered to have the same local spatial energy and variational structure as the original wavelet. The full set of components is used to define the local orientation and variational structure of an analyzed image.

We complement the real wavelet by a special pair of functions, namely the Riesz transform pair [9] of the isotropic wavelet, thus forming a monogenic wavelet [9]. The Riesz pair should be thought of as a single vector valued object that characterizes the orientation of the local variations. If treated as a single vector valued object, the norm of the object may be considered to have the same localization in space and spatial scale as the original radial function, in analog to the Hilbert transform [10] of a 1-D signal. A triplet consisting of the original function plus the Riesz transforms are represented by a positive real-valued amplitude, a direction of variation, and a phase specifying the period of the variation. The triplet may be represented using quaternions [11]. The quaternion algebra, as we shall observe, allows for easy parameterization of phase and orientation structure.

In 1-D analytic wavelets, i.e., Cauchy or Morlet wavelets [12, p. 28], are used to identify local oscillatory structure of a real-valued signal. The monogenic Morse wavelets proposed in this article are a natural 2-D extension of the analytic Morse wavelets [3]. The WT defines a local phase and orientation structure at each spatial position and spatial scale point. To show the properties of the suggested decomposition particular classes of signals are decomposed in terms of the monogenic Morse wavelet decomposition. This includes examples of locally 1-D signal structures such as edges and local oscillations, where general images will correspond to a collection of such phenomena. Note that edges, or spatial discontinuities, have an orientation if they locally correspond to (1-D) curved discontinuities, and will be characterized by their orientation and amplitude. Point (2-D) discontinuities have no associated orientation, and correspond to point locations and amplitudes. We discuss the extraction of these characterising features from the WT of an image.

Oscillations may structurally take the form of 1-D objects such as repeated lines with an even spacing that, if rotated to the appropriate axes, can be locally described as constant in one variable and as a sinusoid in the other. Two-dimensional oscillations, circularly emanating from a single point, when considered locally at a distance from their source may be described approximately as 1-D oscillations. The extraction of such typical signal features in terms of the wavelet coefficients will be discussed. Note that if a sum of oscillatory components is observed, the characterizing features could not be found directly from the monogenic extension of the image, as then a single component with a spatially varying phase function is not an appropriate model for the original image. In this case, it is necessary to combine the calculation of a local phase with scale-localizing methods such as the WT using the monogenic extension of an isotropic real wavelet.

It is well known in image processing that phase information [13] provides important structural information, and scale-local image phase information will enable the disentanglement of different multiscale phase structures present at the same spatial position. Potential applications include image segmentation as 
well as image feature recognition [14], based on criteria defined from the local phase and magnitude via the WT of the observed image. Analyses using the separable discrete WT in addition with local phase structure characterizations, have been previously considered [15], [16]. However, in contrast to such procedures, we define multiple orthogonal continuous wavelets, based on a different 2-D extension to the analytic signal. Other notable quaternionic decompositions includes the work of [17], however this decomposition is only suitable for deterministic images, as the Wigner distribution is not estimated well for signals in noise [18].

The fourth section of the paper deals with application of the theoretical framework to sampled images. We discuss the discrete implementation of the 2-D monogenic Morse WT, via the inverse discrete Fourier transform. We discuss the range of values the scale and spatial parameters the WT may be calculated at from a given discrete sample of the image. Unavoidably, most observed signals are contaminated by noise, and so the local analysis of a noisy image is considered. As the operator problem yields solutions of multiple orthogonal wavelets, uncorrelated estimates of local properties arising from using each of the orthogonal wavelet functions in the analysis may be averaged [1] to achieve a reduced variance in the estimation. Multiple orthogonal filters have been considered in several dimensions for stationary processes [19], and nonstationary processes [20] using the windowed Fourier transform and tensor product windows, but our wavelets are in contrast to these methods orthogonal, nonseparable monogenic wavelet functions. The main developments of this paper are thus concerned with the development of radial localization in 2-D, the introduction of local directional analysis of images via the monogenic wavelets and the derivation of the statistical properties of the developed methods.

\section{NOTATION}

The d-D inner product is given by $\langle g, h\rangle=\int g^{*}(\boldsymbol{x}) h(\boldsymbol{x}) d^{d} \boldsymbol{x}$, and, therefore, the d-D Fourier transform (FT) of $g(\boldsymbol{x})$ is $G(\boldsymbol{f})=\langle\exp (2 \boldsymbol{j} \pi \boldsymbol{f} \cdot \boldsymbol{x}), g\rangle$. The d-D convolution of functions $g_{1}(\boldsymbol{x})$ and $g_{2}(\boldsymbol{x})$ is given by $g_{3}(\boldsymbol{x})=\left(g_{1} \circ g_{2}\right)(\boldsymbol{x})$ with FT $G_{3}(\boldsymbol{f})=G_{1}(\boldsymbol{f}) G_{2}(\boldsymbol{f})$. An arbitrary quaternion is denoted by $q=q_{1}+q_{2} \boldsymbol{i}+q_{3} \boldsymbol{j}+q_{4} \boldsymbol{k}$, where $q_{i} \in \mathbb{R}, i=1, \ldots 4$, $\boldsymbol{i}^{2}=\boldsymbol{j}^{2}=\boldsymbol{k}^{2}=\boldsymbol{i j} \boldsymbol{k}=-1$, while $\boldsymbol{i j}=-\boldsymbol{j i}=\boldsymbol{k}$, $\boldsymbol{i} \boldsymbol{k}=-\boldsymbol{k} \boldsymbol{i}=-\boldsymbol{j}$, and $\boldsymbol{j} \boldsymbol{k}=-\boldsymbol{k} \boldsymbol{j}=\boldsymbol{i}$. For more information on quaternions, see [11]. The d-D FT in terms of any unit quaternion is $G_{\boldsymbol{e}_{q}}(\boldsymbol{f})=\left\langle\exp \left(2 \boldsymbol{e}_{q} \pi \boldsymbol{f} \cdot \boldsymbol{x}\right), g\right\rangle$, so that $G_{\boldsymbol{j}}(\boldsymbol{f}) \equiv G(\boldsymbol{f})$. All implementations are carried out in terms of real-valued quantities. The rotation operation is implemented using matrix $r_{\theta} \equiv[\cos (\theta)-\sin (\theta) ; \sin (\theta) \cos (\theta)]$. We also define the spatial variable $\boldsymbol{x}=[x \cos (\chi), x \sin (\chi)]$, with corresponding Fourier variable $f=[f \cos (\phi), f \sin (\phi)]$, the spatial variable of the local decomposition $\boldsymbol{b}=\left[b \cos \left(\chi_{b}\right), b \sin \left(\chi_{b}\right)\right]$, and its Fourier variable $\boldsymbol{f}_{b}=\left[f_{b} \cos \left(\phi_{b}\right), f_{b} \sin \left(\phi_{b}\right)\right]$, where $x, f, b, f_{b} \geq 0$ and $\chi, \phi, \chi_{b}, \phi_{b} \in[0,2 \pi)$. Let $\mathbb{R}^{+}=\{x: x \in \mathbb{R}, x>0\}$.

\section{LOCALIZATION OPERATORS}

\section{A. 1-D Localization Operators}

One-dimensional local analysis is based on a decomposition of a function $g(t)$ in terms of a set of wavelet functions $\left\{\psi_{a, b}(\cdot)\right\}$, local to scale $a$ and time $b$. The coefficients of this decomposition are defined by (1). Coefficient $w_{\psi}(a, b ; g)$ is thus associated with the function $\psi_{a, b}(t)$. For the coefficients to be interpretable as local contributions of $g(t)$, the mother wavelet $\psi(\cdot)$ is chosen to be well localized to time $t=0$ and frequency $f_{\max }$. In this case $w_{\psi}(a, b ; g)$ is associated with a phase space of time and frequency, and uncovers the behavior of $g(t)$ in this phase space. To obtain well-localized $\psi(\cdot)$, Daubechies and Paul [2] introduced localization operators, and a full discussion of this topic can be found in [5, pp. 17-45].

To construct a localization operator a time-scale region $\mathcal{A}$ and a "fiducial vector" $v^{(1-D, \beta, \gamma)}(t)$ are chosen, where $(\beta, \gamma)$ are given fixed parameters. $v^{(1-D, \beta, \gamma)}(t)$ is picked to be essentially supported near time $t=0$ and frequency $f=f_{0}^{(\beta, \gamma)}(1,0) \neq 0$, and is also the eigenfunction of a suitable differential operator [5, p. 41]. A family of such functions are the Generalised Morse fiducial vectors defined via their FTs in angular frequency $\omega=$ $2 \pi f$ by [3, p. 2663]

$$
V^{(1-D, \beta, \gamma)}(\omega)= \begin{cases}\frac{2^{r / 2+1 / 2} \sqrt{\pi \gamma}}{\sqrt{\Gamma(r)}} \omega^{\beta} e^{-\omega^{\gamma}} & \text { if } \omega>0 \\ 0 & \text { if } \omega<0\end{cases}
$$

where $\gamma \geq 1, \beta>(\gamma-1) / 2$ and $r=(2 \beta+1) / \gamma$. From $v^{(1-D, \beta, \gamma)}(t)$, a family of coherent states $\left\{v_{a, b}^{(1-D, \beta, \gamma)}(t)\right\}$, are defined. Their FTs are given by [3, p. 2663]

$$
\begin{gathered}
V_{a, b}^{(1-D, \beta, \gamma)}(\omega)= \\
|a|^{1 /(2 \gamma)} V^{(1-D, \beta, \gamma)}\left(\left|a^{1 / \gamma-1}\right| a \omega\right) e^{-j b \omega|\omega|^{\gamma-1}}
\end{gathered}
$$

where the parameter $a$ represents a dilation of $v^{(1-D, \beta, \gamma)}(t)$, and when $|a|>1$, the function is stretched out in time. If $\gamma=1$, the time-scale coherent state has been shifted by $b$ in time. If $\gamma \neq 1$, the coherent state has been shifted in time at a different rate at any given scale, or subjected to a generalized time-shift, see [3, p. 2663],[21]. $v_{a, b}^{(1-D, \beta, \gamma)}(t)$ is essentially supported near $t=t_{0}^{(\beta, \gamma)}(a, b)=C_{2}^{(\beta, \gamma)} a^{1 / \gamma-1} b$, and $f=f_{0}^{(\beta, \gamma)}(a, b)=$ $\left(C_{1}^{(\beta, \gamma)} / a^{1 / \gamma}\right)$, where $C_{u}^{(\beta, \gamma)}, u=1,2$ are constants [2, p. 678]. The reason, in this context, for introducing the generalized time-shift is to define projection operators with localization to a larger class of time-frequency regions than solely allowing $\gamma=1$ would permit.

If $v^{(1-D, \beta, \gamma)}(\cdot) \in L^{2}(\mathbb{R})$ is chosen [3, p. 2669] to satisfy the condition $r>1$, then any $g(t) \in L^{2}(\mathbb{R})$ can be reconstructed from the weighted $v_{a, b}^{(1-D, \beta, \gamma)}(t)$, via the "resolution of identity"

$$
g(t)=C_{o}^{1-D} \iint_{\mathbb{R}^{2}}\left\langle v_{a, b}^{(1-D, \beta, \gamma)}, g\right\rangle v_{a, b}^{(1-D, \beta, \gamma)}(t) \frac{d a}{a^{2}} d b
$$

where $C_{o}^{1-D}=(r-1) / 4 \pi$, is a normalization constant. The right-hand side (RHS) of (6) can be thought of as an operator; $g(t)=\mathcal{P}_{\mathbb{R}^{2} ; \beta, \gamma}^{1-D} g(t)$. Instead of reconstructing the function $g(t)$ by acting on it with $\mathcal{P}_{\mathbb{R}^{2} ; \beta, \gamma}^{1-D}$, define an operator $\mathcal{P}_{\mathcal{A} ; \beta, \gamma}^{1 D}$ by restricting the area of the integration to region $\mathcal{A} \subset \mathbb{R}^{2} . \mathcal{P}_{\mathcal{A} ; \beta, \gamma}^{1-D}$ projects the function $g(t)$ into a new function $\mathcal{P}_{\mathcal{A} ; \beta, \gamma}^{1-D} g(t)$, constructed from only using a subset of the family of coherent states, those indexed by $(a, b) \in \mathcal{A}$. Hence, $\mathcal{P}_{\mathcal{A} ; \beta, \gamma}^{1-D} g(t)$ is local to a time-frequency domain $\mathcal{D}^{(\beta, \gamma)}$, determined by $\mathcal{A}$ and $v_{a, b}^{(1-D, \beta, \gamma)}(t)$. An example of $\mathcal{A}$ is the 
Morse region $\mathcal{A}=\mathcal{A}(C)=\left\{(a, b): a^{2}+b^{2}+1 \leq 2 a C\right\}$ [3], where $C$ determines the area of $\mathcal{A}(C)$, denoted $|\mathcal{A}(C)|$. The Morse region, for $C>1$, maps out circles centred at $(a, b)=( \pm C, 0)$ in the time-scale plane, with $|b|<\sqrt{C^{2}-1}$ and $C-\sqrt{C^{2}-1} \leq|a| \leq C+\sqrt{C^{2}-1}$. As $C \rightarrow \infty$, $\mathcal{A}(C) \rightarrow \mathbb{R}^{2}$.

The Morse localization operator $\mathcal{P}_{\mathcal{A}(C) ; \beta, \gamma}^{1-D}$ localizes $g(t)$ to time-frequency region

$$
\mathcal{D}^{(\beta, \gamma)}(C)=\left\{\left(t_{0}^{(\beta, \gamma)}(a, b), f_{0}^{(\beta, \gamma)}(a, b)\right):(a, b) \in \mathcal{A}(C)\right\} .
$$

Specifying $(\beta, \gamma)$ determines $\mathcal{D}^{(\beta, \gamma)}(C)$, the localization region of the projection operator. The same region $\mathcal{A}(C)$ is used to construct different operators, localizing a function to different time-frequency regions by the choice of $(\beta, \gamma)$ and $C$.

The ratio of the energy of a localized function $\mathcal{P}_{\mathcal{A} ; \beta, \gamma}^{1-D} g$ to the original function energy is a measure of the localization of $g(t)$ and is given by $\mu_{g}(\mathcal{D})=\left\langle\mathcal{P}_{\mathcal{A} ; \beta, \gamma}^{1-D} g, \mathcal{P}_{\mathcal{A} ; \beta, \gamma}^{1-D} g\right\rangle /\langle g, g\rangle$. $0 \leq \mu_{g}(\mathcal{D}) \leq 1$, and $g(\cdot)$ is well localized to $\mathcal{D}$ if $\mu_{g}(\mathcal{D})$ is near one. The eigenfunctions of $\mathcal{P}_{\mathcal{A}: \beta, \gamma}^{1-D}$ can be shown to achieve maximum values of $\mu_{g}(\mathcal{D})$ [3], and may be determined from the differential operator that defined the fiducial vector: the localization operator and the differential operator can be shown to commute [5, p. 41].

\section{B. 2-D Localization Operators}

In 2-D the WT coefficients are given by (3), and $w_{\psi}(\boldsymbol{\xi} ; g)$ is associated with a given member of the wavelet family, $\psi_{\boldsymbol{\xi}}(\boldsymbol{x})$. If the mother wavelet is essentially supported at spatial position $\boldsymbol{x}=\mathbf{0}$ and spatial frequency $\boldsymbol{f}=\boldsymbol{f}_{\max }$, then $\psi_{\boldsymbol{\xi}}(\boldsymbol{x})$ is essentially supported at $\boldsymbol{x}=\boldsymbol{b}$ and $\boldsymbol{f}=a^{-1} \boldsymbol{r}_{\theta} \boldsymbol{f}_{\max }$. Then $w_{\psi}(\boldsymbol{\xi} ; g)$ is the local contribution of $g(\boldsymbol{x})$ at $\boldsymbol{x}=\boldsymbol{b}$ and $\boldsymbol{f}=a^{-1} \boldsymbol{r}_{\theta} \boldsymbol{f}_{\text {max }}$. To construct mother wavelets with small essential support, 1-D methods will be mimicked and 2-D localization operators for, in theory, generic spatial and spatial frequency regions $\mathcal{D}_{2}$, are constructed. This is done by specifying a fiducial vector $v(\boldsymbol{x})$, a family of coherent states $\left\{v_{\boldsymbol{\xi}}(\boldsymbol{x})\right\}$, and the local index region, $\mathcal{A}_{2}=\{\boldsymbol{\xi}\}_{\boldsymbol{\xi} \in \mathcal{A}_{2}} \subset \mathbb{R}^{+} \times[0,2 \pi) \times \mathbb{R}^{2} . v_{\boldsymbol{\xi}}(\boldsymbol{x})$ is local to a given spatial position and spatial frequency determined by the value of $\boldsymbol{\xi} .\left\{v_{\boldsymbol{\xi}}(\boldsymbol{x})\right\}_{\boldsymbol{\xi} \in \mathcal{A}_{2}}$ thus specifies a region of space and spatial frequency denoted $\mathcal{D}_{2}$, where the form of $\mathcal{A}_{2}$, combined with the localization of $v_{\boldsymbol{\xi}}(\boldsymbol{x})$, determine its form. The eigenfunctions of the localization operator will be found, and correspond to optimally local functions. The eigenfunctions are appropriate to use as mother wavelets for analysis of images whose local content can be averaged over $\mathcal{D}_{2}$.

Given an arbitrary function $g(\boldsymbol{x}) \in L^{2}\left(\mathbb{R}^{2}\right)$, assuming $v(\boldsymbol{x})$ is square integrable and satisfies the wavelet admissibility condition [7], with $v_{\boldsymbol{\xi}}(\boldsymbol{x})=\mathcal{R}_{\theta} \mathcal{D}_{a} \mathcal{T}_{\boldsymbol{b}} v(\boldsymbol{x})$, a projection operator $\tilde{\mathcal{P}}_{\mathcal{A}_{2}}$ is defined by

$$
\begin{aligned}
\tilde{\mathcal{P}}_{\mathcal{A}_{2}} g(\boldsymbol{x}) & =\tilde{C}_{o} \iiint \int_{\mathcal{A}_{2}}\left\langle v_{\boldsymbol{\xi}}, g\right\rangle v_{\boldsymbol{\xi}}(\boldsymbol{x}) d A_{\boldsymbol{\xi}} \\
d A_{\boldsymbol{\xi}} & =d^{2} \boldsymbol{b} d a a^{-3} d \theta
\end{aligned}
$$

with $\tilde{C}_{o}$ constant. $g(\boldsymbol{x})$ may be reconstructed from the weighted sum of $v_{\boldsymbol{\xi}}(\boldsymbol{x})$ by taking $\mathcal{A}_{2}=\mathbb{R}^{+} \times[0,2 \pi) \times \mathbb{R}^{2}$, or $g(\boldsymbol{x})=$ $\tilde{\mathcal{P}}_{\mathbb{R}^{+} \times[0,2 \pi) \times \mathbb{R}^{2}} g(\boldsymbol{x})[7$, p. 39]. The class of functions achieving large values of the ratio of the energy of the projected signal to the energy of the original signal (denoted $\mu_{g}\left(\mathcal{D}_{2}\right)$ ), are well localized to $\mathcal{D}_{2}$. We shall build a new class of $\tilde{\mathcal{P}}_{\mathcal{A}_{2}}$ by redefining $v_{\boldsymbol{\xi}}(\boldsymbol{x})$ and $\mathcal{A}_{2}$.

If $\psi(\boldsymbol{x})$ is radial, $w_{\psi}(\boldsymbol{\xi} ; g)$ in (3) is formed as a local weighted sum of $g(\boldsymbol{x})$, giving $g(\boldsymbol{x})$ an equal weighting in magnitude at all spatial positions $\boldsymbol{x}$ such that $|\boldsymbol{x}-\boldsymbol{b}|$ takes the same value. This is a desirable property, as the notion of distance in the spatial domain is naturally associated with the Cartesian distance metric. Dahlke and Maass [22] have shown that the orientation and scale may be considered separately when determining well-concentrated 2-D functions; furthermore radial functions have optimal scale versus position localization. Thus isotropically well-localized functions can be modulated in direction to obtain anisotropic well-localized functions. These factors motivate the study of radial localization operators. We define $\mathcal{A}_{2, I}=$ $\{(a, \theta, \boldsymbol{b})\}_{\boldsymbol{\xi} \in \mathcal{A}_{2},} \subset \mathbb{R}^{+} \times[0,2 \pi) \times \mathbb{R}^{2}$ by restricting $\boldsymbol{b}$ in terms of $b=|\boldsymbol{b}|$ and letting $0 \leq \theta<2 \pi$. The subscript of $\mathcal{A}_{2, I}$ indicates the isotropic structure of the region. Denote by $\mathcal{A}_{I}=$ $\{(a, b)\}_{(a, b) \in \mathcal{A}_{I}} \subset \mathbb{R}^{+2}$, the values of $a$ and $b=|\boldsymbol{b}|$ obtained for $\boldsymbol{\xi} \in \mathcal{A}_{2, I}$.

A family of coherent states denoted $\bar{v}_{\xi}^{(\beta, \gamma)}(\boldsymbol{x})$, will be constructed from an isotropic function $v^{(\beta, \gamma)}(\boldsymbol{x})$. A coherent state is (initially) defined by its FT in angular frequency $\omega=2 \pi f$

$$
\begin{aligned}
& \bar{V}_{\boldsymbol{\xi}}^{(\beta, \gamma)}(\boldsymbol{\omega})=a^{1 / \gamma} V^{(\beta, \gamma)}\left(a^{1 / \gamma} \boldsymbol{\omega}\right) e^{-\boldsymbol{j} \omega^{\gamma-1} \boldsymbol{\omega} \cdot \boldsymbol{b}}, \\
& V^{(\beta, \gamma)}(\boldsymbol{\omega})=\omega^{-1 / 2+\gamma / 2} V^{(1-D, \beta, \gamma)}(\omega)
\end{aligned}
$$

where $V^{(1-D, \beta, \gamma)}(\omega)$ is given by (4). The scale parameter $a$ for $a>1$ stretches $v^{(\beta, \gamma)}(\boldsymbol{x})$ isotropically in the spatial domain. The region $\mathcal{A}_{2, I}$ does not restrict the value of $\theta$ and $v^{(\beta, \gamma)}(\boldsymbol{x})$ is assumed to be radial: thus the choice of localization in $\theta$ is redundant, and is not used in this section. In 2-D, the coherent state is defined with a generalized spatial shift, in analog with (5). The generalized shift is introduced to permit the localization to a larger class of localization regions, as in 1-D. $V^{(\beta, \gamma)}(\boldsymbol{\omega})$ is essentially supported only at a limited set of frequencies and so the multiplication by the complex exponential is interpreted roughly as an ordinary spatial shift. Using (7) to define the projection operator, replacing $v_{\boldsymbol{\xi}}(\boldsymbol{x})$ by $\bar{v}_{\boldsymbol{\xi}}^{(\beta, \gamma)}(\boldsymbol{\omega})$ and $\mathcal{A}_{2}$ by $\mathcal{A}_{2, I}$, a projection of the function $g(\boldsymbol{x})$ is defined, and denoted $\overline{\mathcal{P}}_{\mathcal{A}_{2, I} ; \beta, \gamma} g(\boldsymbol{x})$. As we wish to obtain well-localized isotropic functions, the next step is to determine the isotropic eigenfunctions of this localization operator.

\section{Isotropic Localization of Isotropic Functions}

Here it is assumed that $g_{I}(\cdot)$ is isotropic. We start by simplifying the expression for $\overline{\mathcal{P}}_{\mathcal{A}_{2, I} ; \beta, \gamma} g_{I}(\boldsymbol{x})$ for such $g_{I}(\boldsymbol{x})$. This will lead to the definition of a new operator, valid for isotropic functions only. The act of projecting $g_{I}(\boldsymbol{x})$ corresponds to integrating $\left\langle\bar{v}_{\boldsymbol{\xi}}^{(\beta, \gamma)}, g_{I}\right\rangle \bar{v}_{\boldsymbol{\xi}}^{(\beta, \gamma)}$ over $\mathcal{A}_{2, I}$, and the projection is calculated in the frequency domain. Write $\left\langle\bar{v}_{\boldsymbol{\xi}}^{(\beta, \gamma)}, g_{I}\right\rangle=(2 \pi)^{-2} \iint_{\mathbb{R}^{2}} d^{2} \boldsymbol{\omega}_{2}$

$\bar{V}_{\boldsymbol{\xi}}^{(\beta, \gamma) *}\left(\boldsymbol{\omega}_{2}\right) G_{I}\left((2 \pi)^{-1} \boldsymbol{\omega}_{2}\right)$, where $\boldsymbol{\omega}_{2}=\left[\omega_{2 ; 1} \omega_{2 ; 2}\right]^{T}$, and let $\phi_{2}=\tan ^{-1}\left(\omega_{2 ; 2} / \omega_{2 ; 1}\right)$, as well as $\chi_{b}=\tan ^{-1}\left(b_{2} / b_{1}\right)$. Denote the zeroth Bessel function by $J_{0}(x)$ [23, pp. 358-362], 
and recall $b=|\boldsymbol{b}|, \omega_{2}=\left|\boldsymbol{\omega}_{2}\right|$. The FT of (7) with $v_{\boldsymbol{\xi}}(\cdot)=$ $\bar{v}_{\xi}^{(\beta, \gamma)}(\cdot), \mathcal{A}_{2}=\mathcal{A}_{2, I}, \tilde{C}_{o}=\bar{C}_{o}$ and $d A_{\xi_{I}}=b d b d a a^{-3}$ is

$$
\begin{aligned}
\overline{\mathcal{P}}_{\mathcal{A}_{2, I} ; \beta, \gamma} G_{I}\left(\frac{\boldsymbol{\omega}}{2 \pi}\right) \\
=\frac{\bar{C}_{o}}{(2 \pi)^{2}} \iiint \int_{\mathcal{A}_{2, I}} \iint_{\mathbb{R}^{2}} \bar{V}_{\boldsymbol{\xi}}^{(\beta, \gamma)}(\boldsymbol{\omega}) \\
\quad \times \bar{V}_{\boldsymbol{\xi}}^{(\beta, \gamma) *}\left(\boldsymbol{\omega}_{2}\right) G_{I}\left(\frac{\boldsymbol{\omega}_{2}}{2 \pi}\right) d^{2} \boldsymbol{\omega}_{2} d A_{\boldsymbol{\xi}} \\
=2 \pi \bar{C}_{o} \iint_{\mathcal{A}_{I}} \int_{\mathbb{R}^{+}} V_{\boldsymbol{\xi}_{I}}^{(2, \beta, \gamma) *}\left(\omega_{2}\right) G_{I}\left(\frac{\boldsymbol{\omega}_{2}}{2 \pi}\right) \\
\quad \times V_{\boldsymbol{\xi}_{I}}^{(2, \beta, \gamma)}(\omega) \omega_{2} d \omega_{2} d A_{\boldsymbol{\xi}_{I}} \\
=\bar{C}_{o} \iint_{\mathcal{A}_{I}} \int_{\mathbb{R}^{+}} \kappa\left(\omega, \omega_{2} ; a, b\right) \frac{d \omega_{2} d b d a}{a^{2}}
\end{aligned}
$$

thus, defining $\kappa(\cdot)$, and the angular average of the coherent state is $V_{\boldsymbol{\xi}_{I}}^{(2, \beta, \gamma)}(\omega)=\bar{V}_{(a, 0,0)}^{(\beta, \gamma)}(\boldsymbol{\omega}) J_{0}\left(\omega^{\gamma} b\right)$ with $\boldsymbol{\xi}_{I}=[a, b]$. A change of variables to polar coordinates and integration over $\phi_{2}, \theta$ and $\chi_{b}$ have been implemented, note that $d^{2} \boldsymbol{b} d^{2} \boldsymbol{\omega}_{2}=\omega_{2} d \omega_{2} d \phi_{2} b d b d \chi_{b}$, and the integration of the complex exponential follows by [23, p. 364, 8.2.1]. The term $a$ represents scale but $\boldsymbol{\xi}_{I}$ does not depend on $\chi_{b}$ or $\theta$. When projecting $g_{I}(\boldsymbol{x})$ the $\boldsymbol{b}$ parameter in (9) corresponds to a generalized spatial shift and the $b$ parameter in (10) is the distance of the generalized spatial shift from the origin. As $g_{I}(\boldsymbol{x})$ is radial, the orientation of the shift is not important when calculating the projection over $0 \leq \theta, \chi_{b}<2 \pi$.

To project a radial function the expression in (9) can therefore be simplified to (10). It is still very difficult to determine the radial eigenfunctions of the operator from (10). We shall define another localization operator that approximately performs the same localization as $\overline{\mathcal{P}}_{\mathcal{A}_{2, I} ; \beta, \gamma}$, when $g_{I}(\boldsymbol{x})$ is radial. The action of the inconvenient Bessel function in (10) will be approximated, and $v_{\boldsymbol{\xi}_{I}}^{(2, \beta, \gamma)}(\boldsymbol{x})$ will be replaced by another function, this defining the new operator.

We define an alternative angular average coherent state $v_{\boldsymbol{\xi}_{I}}^{(1, \beta, \gamma)}(\boldsymbol{x})$, for $a>0$ and $b>0$ in the angular frequency domain by

$$
\begin{array}{r}
V_{\boldsymbol{\xi}_{I}}^{(1, \beta, \gamma)}(\boldsymbol{\omega})=a^{1 / \gamma}\left(a^{1 / \gamma} \omega\right)^{-1 / 2+\gamma / 2} V^{(1-D, \beta, \gamma)}\left(a^{1 / \gamma} \omega\right) \\
\times \frac{\cos \left(\omega^{\gamma} b-\frac{\pi}{4}\right)}{\sqrt{2^{-1} \pi \omega^{\gamma} b}} .
\end{array}
$$

$V^{(1-D, \beta, \gamma)}(\cdot)$ is defined by (4) and $\omega=2 \pi f . v_{\xi_{I}}^{(1, \beta, \gamma)}(\boldsymbol{x})$ is well localized in frequency near the circle $|\boldsymbol{f}|=f_{0}$, where $f_{0}=\left|\arg \max \omega^{-1 / 2} V^{(1-D, \beta, \gamma)}(\omega)\right| \times(2 \pi)^{-1}$, and it decays isotropically in space from $\boldsymbol{x}=0$. The $V_{\xi_{I}}^{(1-D, \beta, \gamma)}(\cdot)$ are localized to the circle $|\boldsymbol{f}|=f_{0}^{(\beta-1 / 2, \gamma)} / a^{1 / \gamma}$. The factor $\left(a^{1 / \gamma} \omega\right)^{-1 / 2+\gamma / 2}$ in the coherent state is added to ensure a suitable normalization of the function defined by (11).

To clarify the effects of replacing $v_{\boldsymbol{\xi}_{I}}^{(2, \beta, \gamma)}(\boldsymbol{x})$ by $v_{\boldsymbol{\xi}_{I}}^{(1, \beta, \gamma)}(\boldsymbol{x})$ in (10) we introduce $T_{b}^{(1)}(\omega, b)=\cos \left(\omega^{\gamma} b-\pi / 4\right) / \sqrt{2^{-1} \pi \omega^{\gamma} b}$, and $T_{b}^{(2)}(\omega, b)=J_{0}\left(\omega^{\gamma} b\right)$. The angular average of the translation by $\boldsymbol{b}$, denoted by $T_{b}^{(2)}(\omega, b)$, has a decay for large values of $\omega^{\gamma} b$ that is the same as the decay of $T_{b}^{(1)}(\omega, b)$. Their zero-crossing structure is also similar, and replacing one by the other may be justified in terms of the asymptotic expansion of the Bessel function [24]. Ignoring small arguments, the two functions are performing a similar action, but the functional behavior for small values of $\omega$ of $T_{b}^{(2)}(\omega, b)$ and $T_{b}^{(1)}(\omega, b)$ are respectively given by $T_{b}^{(2)}(\omega, b)=1+O\left(\omega^{2 \gamma}\right)$ and $T_{b}^{(1)}(\omega, b)=1 / \sqrt{\pi \omega^{\gamma} b}+O\left(\omega^{\gamma / 2}\right)$, where the latter is unbounded near $\omega \rightarrow 0$. The angular average of the coherent state defined using $T_{b}^{(u)}(\omega, b)$ is written as $V_{\boldsymbol{\xi}_{I}}^{(u ; \beta, \gamma)}(\boldsymbol{\omega})$ and for small values of $\omega$

$$
\begin{aligned}
V_{\boldsymbol{\xi}_{I}}^{(u ; \beta, \gamma)}(\boldsymbol{\omega})= & \sqrt{\frac{2^{r+1} \pi \gamma}{\Gamma(r)}} a^{1 / \gamma}\left(a^{1 / \gamma} \omega\right)^{-1 / 2+\gamma / 2}\left(a^{1 / \gamma} \omega\right)^{\beta} \\
& \times\left[1+O\left(\omega^{\gamma}\right)\right] T_{b}^{(u)}(\omega, b) \\
V_{\boldsymbol{\xi}_{I}}^{(1 ; \beta, \gamma)}(\boldsymbol{\omega})= & E_{1} \omega^{\beta-1 / 2}+O\left(\omega^{-1 / 2+\gamma+\beta}\right) \\
V_{\boldsymbol{\xi}_{I}}^{(2 ; \beta, \gamma)}(\boldsymbol{\omega})= & E_{2} \omega^{\beta+\gamma / 2-1 / 2}+O\left(\omega^{\gamma}\right)
\end{aligned}
$$

for some constant $E_{1}$ and $E_{1}$. Hence, for $|\omega| \rightarrow 0$, both $V_{\boldsymbol{\xi}_{I}}^{(u ; \beta, \gamma)}(\boldsymbol{\omega}) \approx 0$ as long as $\beta>1 / 2$, combined with the previous constraints of $\gamma \geq 1$, and $\beta>(\gamma-1) / 2$, [3, p. 2663]. Given that the two different operators $T_{b}^{(1)}$ and $T_{b}^{(2)}$ take approximately the same value at all values $\boldsymbol{\omega}$ for which $V_{\boldsymbol{\xi}_{I}}^{(u ; \beta, \gamma)}(\boldsymbol{\omega})$ has nonnegligible magnitude, $v_{\boldsymbol{\xi}_{I}}^{(1 ; \beta, \gamma)}(\boldsymbol{x})$ can be given the same interpretation as $v_{\boldsymbol{\xi}_{I}}^{(2 ; \beta, \gamma)}(\boldsymbol{x})$.

Given the similar nature of $v_{\boldsymbol{\xi}_{I}}^{(2 ; \beta, \gamma)}(\boldsymbol{x})$ and $v_{\boldsymbol{\xi}_{I}}^{(1 ; \beta, \gamma)}(\boldsymbol{x})$ we shall replace the former function by the latter in (10) and, thus, define a new projection operator. We shall write $v_{\boldsymbol{\xi}_{I}}^{(\beta, \gamma)}(\boldsymbol{x})$ for $\sqrt{2 \pi} v_{\boldsymbol{\xi}_{I}}^{(1 ; \beta, \gamma)}(\boldsymbol{x})$, and use this as the "radial coherent state." A projection operator is then defined for any radial function $g_{I}(\boldsymbol{x})$ in terms of an inner product $\left\langle g_{1}, g_{2}\right\rangle_{I}=(1 / 2 \pi)\left\langle G_{1}, G_{2}\right\rangle_{I}=$ $\int_{0}^{\infty} G_{1}^{*}(f) G_{2}(f) f d f$ and localization region $\mathcal{A}_{I}$. We take $\mathcal{A}_{I}=$ $\mathcal{A}^{+}(C)=\left\{(a, b): a^{2}+b^{2}+1 \leq 2 a C, b>0\right\}$, where the + is added to the notation as $\mathcal{A}^{+}(C)$ is equivalent to a quarter of the Morse domain $\mathcal{A}(C)$. $C$ regulates the area of $\mathcal{A}^{+}(C)$, denoted $\left|\mathcal{A}^{+}(C)\right| \cdot(\beta, \gamma)$ determine the shape of the localization domain

$$
\begin{aligned}
\mathcal{D}^{(+; \beta, \gamma)}(C)= & \left\{(\boldsymbol{x}, \boldsymbol{f}), f=C_{3}^{(\beta, \gamma)} a^{-1 / \gamma},\right. \\
& \left.x_{l}=C_{4}^{(\beta, \gamma)} b_{l} a^{1 / \gamma-1}, l=1,2,(a, b) \in \mathcal{A}^{+}(C)\right\}
\end{aligned}
$$

for $C_{u}^{(\beta, \gamma)}, u=3,4$ constants. $C_{u}^{(\beta, \gamma)}, u=3,4$ are the average spatial position and modulus of the spatial frequency of the coherent state whose angular average corresponds to $v_{(1,0)}^{(2 ; \beta, \gamma)}(\boldsymbol{x})$.

The projection operator, for a radial $g_{I}(\boldsymbol{x})$, is then defined by

$$
\begin{aligned}
\mathcal{P}_{\mathcal{A}^{+}(C) ; \beta, \gamma}\left\{G_{I}\right\}\left(\frac{\boldsymbol{\omega}_{1}}{2 \pi}\right) \\
=2 \pi C_{o} \int_{\mathcal{A}^{+}(C)} V_{\boldsymbol{\xi}_{I}}^{(\beta, \gamma)}\left(\boldsymbol{\omega}_{1}\right)\left\langle V_{\boldsymbol{\xi}_{I}}^{(\beta, \gamma)}, G_{I}\right\rangle_{I} \frac{d a}{a^{3}} b d b \\
\quad \times \mathcal{P}_{\mathcal{A}^{+}(C) ; \beta, \gamma}\left\{G_{I}\right\}\left(\frac{\boldsymbol{\omega}_{1}}{2 \pi}\right)
\end{aligned}
$$




$$
\begin{aligned}
= & C_{o} \int_{\omega_{2}=0}^{\infty} \iint_{a^{2}+b^{2}+1 \leq 2 a C} \kappa_{2}\left(\omega_{1}, \omega_{2} ; a, b\right) \frac{d a}{a^{2}} d b d \omega_{2} \\
& \times \kappa_{2}\left(\omega_{1}, \omega_{2} ; a, b\right) \\
= & 2 a^{1 / \gamma} V^{(1-D, \beta, \gamma)}\left(a^{1 / \gamma} \omega_{1}\right) \\
& \times \sqrt{\frac{\omega_{2}}{\omega_{1}}} V^{(1-D, \beta, \gamma) *}\left(a^{1 / \gamma} \omega_{2}\right) \\
\times & G_{I}\left(\frac{\omega_{2}}{2 \pi}\right) \cos \left(\left(\omega_{1}^{\gamma}-\omega_{2}^{\gamma}\right) b\right) .
\end{aligned}
$$

To retrieve the spatial domain operator the inverse FT of (13) is calculated, and note that by definition $\omega_{1}>0$. If the family $\left\{v_{\boldsymbol{\xi}_{I}}^{(2, \beta, \gamma)}(\boldsymbol{x})\right\}$ rather than $\left\{v_{\boldsymbol{\xi}_{I}}^{(1, \beta, \gamma)}(\boldsymbol{x})\right\}$ were to be used to define the operator, then (12) is equivalent to (10), with $C_{o}$ replaced by $\bar{C}_{o}$.

It follows that

$$
\begin{aligned}
G_{I}\left(\frac{\boldsymbol{\omega}_{1}}{2 \pi}\right)= & C_{o} \int_{\omega_{2}=0}^{\infty} \int_{a=0}^{\infty} \int_{b=0}^{\infty} 2 a^{1 / \gamma} V^{(1-D, \beta, \gamma)}\left(a^{1 / \gamma} \omega_{1}\right) \\
& \times \sqrt{\frac{\omega_{2}}{\omega_{1}}} V^{(1-D, \beta, \gamma) *}\left(a^{1 / \gamma} \omega_{2}\right) G_{I}\left(\frac{\boldsymbol{\omega}_{2}}{2 \pi}\right) \\
& \times \cos \left(\left(\omega_{1}^{\gamma}-\omega_{2}^{\gamma}\right) b\right) \frac{d a}{a^{2}} d b d \omega_{2} \\
= & C_{o} \int_{\omega_{2}=0}^{\infty} \int_{a=0}^{\infty} \int_{b=-\infty}^{\infty} a^{1 / \gamma} V^{(1-D, \beta, \gamma)}\left(a^{1 / \gamma} \omega_{1}\right) \\
& \times \sqrt{\frac{\omega_{2}}{\omega_{1}}} V^{(1-D, \beta, \gamma) *}\left(a^{1 / \gamma} \omega_{2}\right) \\
& \times G_{I}\left(\frac{\boldsymbol{\omega}_{2}}{2 \pi}\right) \cos \left(\left(\omega_{1}^{\gamma}-\omega_{2}^{\gamma}\right) b\right) \frac{d a}{a^{2}} d b d \omega_{2} \\
= & C_{o} 2(2 \pi)^{2} G_{I}\left(\frac{\boldsymbol{\omega}_{1}}{2 \pi}\right) \frac{1}{r-1} .
\end{aligned}
$$

A "resolution of identity" (cf. [3, p. 2669]) is thus achieved for radial functions when $C_{o}=(r-1) /\left(2^{3} \pi^{2}\right)$. The radial function is reconstructed as the domain encompasses all space for $\boldsymbol{\xi}$.

The eigenfunctions of the operator defined in (12) can be found by solving

$$
\mathcal{P}_{\mathcal{A}^{+}(C) ; \beta, \gamma}\left\{G_{I}\right\}\left(\frac{\boldsymbol{\omega}_{1}}{2 \pi}\right)=\lambda G_{I}\left(\frac{\boldsymbol{\omega}_{1}}{2 \pi}\right) .
$$

Fortunately, this equation can be directly related to the corresponding 1-D problem whose solution is known. The 1-D Morse wavelet projection operator can for $\psi(\cdot)$ real and even, for $\omega_{1}>0$, (similar expressions are derived for $\omega_{1}<0$, but as we shall use this to obtain solutions to (15) we only need to consider $\omega_{1}>0,(a>0)$ and the $\sin (\cdot)$ term vanishes due to symmetry arguments)

$$
\begin{aligned}
& \mathcal{P}_{\mathcal{A}(C) ; \beta, \gamma}^{1-D}\{\Psi\}\left(\frac{\omega_{1}}{2 \pi}\right) \\
&=C_{o}^{1-D} \int_{\omega_{2}=0}^{\infty} \iint_{a^{2}+b^{2}+1 \leq 2 a C, a \in \mathbb{R}^{+}, b \in \mathbb{R}} \frac{d a}{a^{2}} d b \frac{d \omega_{2}}{2 \pi} \\
& \quad \times a^{1 / \gamma} V^{(1-D, \beta, \gamma)}\left(a^{1 / \gamma} \omega_{1}\right) V^{(1-D, \beta, \gamma) *}\left(a^{1 / \gamma} \omega_{2}\right) \Psi\left(\frac{\omega_{2}}{2 \pi}\right) \\
& \quad \times \cos \left[b\left(\omega_{1}^{\gamma}-\omega_{2}^{\gamma}\right)\right] \\
&= \frac{C_{o}^{1-D}}{2 \pi} \int_{\omega_{2}=0}^{\infty} \iint_{a^{2}+b^{2}+1 \leq 2 a C, a \in \mathbb{R}^{+}, b \in \mathbb{R}^{+}} \frac{d a}{a^{2}} d b d \omega_{2} \\
& \times \kappa_{1}\left(\omega_{1}, \omega_{2}, a, b\right)
\end{aligned}
$$

where we define

$$
\begin{aligned}
& \kappa_{1}\left(\omega_{1}, \omega_{2} ; a, b\right)=2 a^{1 / \gamma} V^{(1-D, \beta, \gamma)}\left(a^{1 / \gamma} \omega_{1}\right) \\
& \quad \times V^{(1-D, \beta, \gamma)}\left(a^{1 / \gamma} \omega_{2}\right) \Psi\left(\frac{\omega_{2}}{2 \pi}\right) \cos \left(\left(\omega_{1}^{\gamma}-\omega_{2}^{\gamma}\right) b\right) .
\end{aligned}
$$

Kernel $\kappa_{1}(\cdot, \cdot ; \cdot, \cdot)$ in (17) is similar to kernel $\kappa_{2}(\cdot, \cdot ; \cdot, \cdot)$ of $(13)$, the only difference being that $\kappa_{2}(\cdot, \cdot ; \cdot, \cdot)$ has the extra term, $\sqrt{\omega_{2} / \omega_{1}}$, and $C_{o}^{1-D}=(r-1) /(4 \pi)$. The Morse wavelets [3] are the solution to

$$
\mathcal{P}_{\mathcal{A}(C) ; \beta, \gamma}^{1-D}\left\{\Psi^{1-D}\right\}\left(\frac{\omega_{1}}{2 \pi}\right)=\lambda^{1-D} \Psi^{1-D}\left(\frac{\omega_{1}}{2 \pi}\right) .
$$

Consider (15); multiply both sides by $\sqrt{\omega_{1} /(2 \pi)}$, set $G_{I}(\omega) \sqrt{\omega}=\Psi^{1-D}(\omega)$, and note that the equation to be solved has now exactly the form of (18)

$$
\begin{aligned}
\sqrt{\frac{\omega_{1}}{2 \pi}} \mathcal{P}_{\mathcal{A}^{+}(C) ; \beta, \gamma}\left\{G_{I}\right\}\left(\frac{\omega_{1}}{2 \pi}\right) & =\frac{2 \pi C_{o} \mathcal{P}_{\mathcal{A}(C) ; \beta, \gamma}^{1-D}\left\{\Psi^{1-D}\right\}\left(\frac{\omega_{1}}{2 \pi}\right)}{C_{o}^{1-D}} \\
& =\lambda^{1-D} \Psi^{1-D}\left(\frac{\omega_{1}}{2 \pi}\right)
\end{aligned}
$$

where $2 \pi C_{o} / C_{o}^{1-D}=1$, is used. Thus, the solutions of (15) are given by

$$
\Psi_{n ; l, m}^{(e)}(\boldsymbol{f})=\Psi_{n ; l, m}^{(e)}(f)=\frac{1}{\sqrt{f}} \Psi_{n ; \beta, \gamma}^{(1-D, e)}(f), f>0
$$

for $n=0,1, \ldots$, where $\Psi_{n ; \beta ; \gamma}^{(1-D, e)}(\cdot)$ are the even Morse wavelets defined in 1-D, see [3], $l=\beta-(1 / 2), m=\gamma$, and $n \in \mathbb{N}$ enumerates the eigenvectors. The eigenvalues correspond to

$$
\begin{aligned}
\lambda_{n, r}(C)= & \lambda_{n, r}^{1-D} \\
= & \frac{\Gamma(r+n)}{\Gamma(n+1) \Gamma(r-1)} \\
& \times \int_{0}^{(C-1 / C+1)} x^{n}(1-x)^{r-2} d x
\end{aligned}
$$

and this yields the concentration of

$$
\begin{array}{r}
\mu_{\psi_{n ; l, m}^{(e)}}\left(\mathcal{D}^{(+; \beta, \gamma)}(C)\right)=\left\langle\mathcal{P}_{\mathcal{A}^{+}(C) ; \beta, \gamma} \psi_{n ; l, m}^{(e)}, \mathcal{P}_{\mathcal{A}^{+}(C) ; \beta, \gamma} \psi_{n ; l, m}^{(e)}\right\rangle \\
\times\left\langle\psi_{n ; l, m}^{(e)}, \psi_{n ; l, m}^{(e)}\right\rangle^{-1}=\lambda_{n, r}^{2}(C)
\end{array}
$$

decreasing monotonically in $n$. It may seem surprising that, in 2-D, the same eigenvalues, and, thus, concentration values, are found as in the 1-D case. Note that the result is only derived for a radial $g_{I}(\boldsymbol{x})$, that is constrained to the same behavior in both spatial directions. The hypervolume of $\mathcal{D}^{(+; \beta, \gamma)}(C)$ is directly related to $C$, and we may formulate the notion of bias in the estimation (leakage) of local properties of the signal in terms of the eigenvalues, as $1-\lambda_{n, r}^{2}(C)$ [3]. To reconstruct a single function $g(\boldsymbol{x})$, the wavelet $\psi_{n ; l, m}^{(e)}(\boldsymbol{x})$ can, for a fixed value of $n$, be used to calculate $w_{\psi_{n ; l, m}^{(e)}}(\boldsymbol{\xi} ; g)$ and $g(\boldsymbol{x})$ can be reconstructed from (7) with $v_{\boldsymbol{\xi}}(\boldsymbol{x})=\psi_{n ; l, m, \boldsymbol{\xi}}^{(e)}(\boldsymbol{x})$. In theory functions may also be represented by a decomposition in terms of the full set $\left\{\psi_{n ; l, m}^{(e)}(\boldsymbol{x})\right\}_{n}$, as this will form a basis of the radial square integrable functions. The spatial localization properties of the 
Morse wavelets deteriorate with increasing $n$ and, therefore, following [1], only a finite number $N$ will be used for analysis.

\section{Nonradial Localization Operators}

$\mathcal{P}_{\mathcal{A}^{+}(C) ; \beta, \gamma}$ was constructed in the radial frequency domain and is valid for $g_{I}(\boldsymbol{x})$. To define an operator in 2-D, a generic region $\mathcal{A}_{2}$ will be used, and define $\left|\mathcal{A}_{2}\right|$ as the hypervolume of $\mathcal{A}_{2}$. Let

$\mathcal{A}_{2}^{+}(C)=\left\{\boldsymbol{\xi}: a^{2}+b^{2}+1 \leq a C, 0 \leq \theta \leq 2 \pi, \boldsymbol{b} \in \mathbb{R}^{2}, a>0\right\}$

i.e., the region corresponds to a radial domain, with no directional asymmetries. The scale and modulus of the spatial shift are constrained like the $(a, b)$ of the Morse region, $\mathcal{A}^{+}(C)$. A coherent state $\tilde{v}_{\xi}^{(\beta, \gamma)}(\boldsymbol{x})$ will be constructed to form the building block of the projection operator. The coherent state at $\xi$ at a given angular frequency $\boldsymbol{\omega}=2 \pi f$ is defined from generic fiducial vector $\tilde{v}^{(\beta, \gamma)}(\boldsymbol{x})$ by

$$
\tilde{V}_{\boldsymbol{\xi}}^{(\beta, \gamma)}(\boldsymbol{\omega})=a^{1 / \gamma} \tilde{V}^{(\beta, \gamma)}\left(a^{1 / \gamma} \boldsymbol{r}_{-\theta} \boldsymbol{\omega}\right) e^{-\boldsymbol{j} \boldsymbol{b} \cdot \boldsymbol{\omega} \boldsymbol{\omega}^{\gamma-1}} .
$$

The definition of $\tilde{v}_{\boldsymbol{\xi}}^{(\beta, \gamma)}(\boldsymbol{x})$ is analogous to (2), and corresponds to (8) if $\tilde{v}^{(\beta, \gamma)}=\bar{v}^{(\beta, \gamma)} \cdot \tilde{v}_{\boldsymbol{\xi}}^{(\beta, \gamma)}(\boldsymbol{x})$ is a dilation of $\tilde{v}^{(\beta, \gamma)}(\boldsymbol{x})$ by $a^{1 / \gamma}$, the translation is a generalized spatial shift in both axes, i.e., the coherent state is multiplied by $e^{-\boldsymbol{j} \boldsymbol{b} \cdot \boldsymbol{\omega} \omega^{\gamma-1}}$, and there is a rotation of $\theta$. The localization operator $\tilde{\mathcal{P}}_{\mathcal{A}_{2} ; \beta, \gamma}$ acting on any function $g(\boldsymbol{x}) \in L^{2}\left(\mathbb{R}^{2}\right)$ is defined from (7) with $v_{\boldsymbol{\xi}}(\boldsymbol{x})=$ $\tilde{v}_{\xi}^{(\beta, \gamma)}(\boldsymbol{x})$, given by (21) and an arbitrary $\mathcal{A}_{2} . \tilde{\mathcal{P}}_{\mathcal{A}_{2} ; \beta, \gamma}$ localizes a function $g(\cdot)$ over a region $\mathcal{D}_{2}$. By calculating the moments of $\tilde{v}_{\boldsymbol{\xi}}^{(\beta, \gamma)}$ in the spatial and spatial frequency domains we determine that by restricting $\boldsymbol{\xi}$ to $\mathcal{A}_{2}$ we restrict $\tilde{\mathcal{P}}_{\mathcal{A}_{2} ; \beta, \gamma} g(\boldsymbol{x})$ to some spatial and spatial frequency region $\mathcal{D}_{2}$. If $\mathcal{A}_{2}=\mathcal{A}_{2}^{+}(C)$ and $\tilde{v}^{(\beta, \gamma)}(\cdot)=\bar{v}^{(\beta, \gamma)}(\cdot)$ then

$$
\begin{aligned}
\mathcal{D}_{2}=\mathcal{D}_{2}^{(\beta, \gamma)}(C)= & \left\{(\boldsymbol{x}, f), f=C_{3}^{(\beta, \gamma)} a^{-1 / \gamma},\right. \\
& x_{l}=C_{4}^{(\beta, \gamma)} b_{l} a^{1 / \gamma-1}, \\
& \left.l=1,2,(a, b) \in \mathcal{A}^{+}(C)\right\} \equiv \mathcal{D}^{(+; \beta, \gamma)}(C)
\end{aligned}
$$

for $C_{u}^{(\beta, \gamma)}, u=3,4$, taking the same values as in Section III-C.

We need to normalize the operator and take $\tilde{C}_{o}$ such that as $\left|\mathcal{A}_{2}\right| \longrightarrow \infty, \tilde{\mathcal{P}}_{\mathcal{A}_{2} ; \beta, \gamma}\{g\}(x) \rightarrow g(x)$. With changes of variable given by $\boldsymbol{s}_{2}^{q}=s_{2}^{q}\left[\cos \left(\phi_{2}\right) \sin \left(\phi_{2}\right)\right]$, $\boldsymbol{a}_{2}=a_{2}\left[\cos \left(\theta_{2}\right) \sin \left(\theta_{2}\right)\right], s_{2}=\omega^{\gamma}, a_{2}=a^{1 / \gamma} \omega, \theta_{2}=\phi-\theta$, and $q \in \mathbb{R}$

$$
\begin{aligned}
G\left(\frac{\boldsymbol{\omega}}{2 \pi}\right)= & \frac{\tilde{C}_{o}}{(2 \pi)^{2}} \int_{-\infty}^{\infty} \int_{-\infty}^{\infty} \int_{0}^{2 \pi} \int_{-\infty}^{\infty} \int_{-\infty}^{\infty} \int_{0}^{\infty} d A_{\boldsymbol{\xi}} \\
& \times d^{2} \boldsymbol{\omega}_{2} \tilde{V}_{\boldsymbol{\xi}}^{(\beta, \gamma)}(\boldsymbol{\omega}) \tilde{V}_{\boldsymbol{\xi}}^{(\beta, \gamma) *}\left(\boldsymbol{\omega}_{2}\right) G\left(\frac{\boldsymbol{\omega}_{2}}{2 \pi}\right) \\
= & \frac{\tilde{C}_{o}}{(2 \pi)^{2}} \int_{-\infty}^{\infty} \int_{-\infty}^{\infty} \int_{0}^{2 \pi} \int_{-\infty}^{\infty} \int_{-\infty}^{\infty} \int_{0}^{\infty} d A_{\boldsymbol{\xi}} \\
& \times a^{2 / \gamma} \tilde{V}^{(\beta, \gamma)}\left(a^{1 / \gamma} \boldsymbol{r}_{-\theta} \boldsymbol{\omega}\right) e^{-\boldsymbol{j} \boldsymbol{b}\left(\omega^{\gamma-1} \boldsymbol{\omega}-\boldsymbol{s}_{2}\right)} \\
& \times \tilde{V}^{(\beta, \gamma) *}\left(a^{1 / \gamma} \boldsymbol{r}_{-\theta} \boldsymbol{s}_{2}^{1 / \gamma}\right) \\
& \times G\left(\frac{s_{2}^{1 / \gamma}[\cos (\phi) \sin (\phi)]^{T}}{2 \pi}\right) \frac{\omega_{2}^{2}}{\gamma} \omega_{2}^{-2 \gamma} \\
& \times s_{2} d s_{2} d \phi_{2}
\end{aligned}
$$

$$
\begin{aligned}
= & \tilde{C}_{o} \int_{-\infty}^{\infty} \int_{-\infty}^{\infty} \int_{0}^{2 \pi} \int_{0}^{\infty} \frac{d a}{a^{3}} d \theta d^{2} \boldsymbol{s}_{2} a^{2 / \gamma} \\
& \times \delta\left(\omega^{\gamma-1} \boldsymbol{\omega}-\boldsymbol{s}_{2}\right) \tilde{V}^{(\beta, \gamma)}\left(a^{1 / \gamma} \boldsymbol{r}_{-\theta} \boldsymbol{\omega}\right) \\
& \times \tilde{V}^{(\beta, \gamma) *}\left(a^{1 / \gamma} \boldsymbol{r}_{-\theta} \boldsymbol{s}_{2}^{1 / \gamma}\right) G\left(\frac{\boldsymbol{s}_{2}^{1 / \gamma}}{2 \pi}\right) \frac{1}{\gamma} s_{2}^{-2+2 / \gamma} \\
= & \tilde{C}_{o} G\left(\frac{\boldsymbol{\omega}}{2 \pi}\right) \int_{-\infty}^{\infty} \int_{-\infty}^{\infty} \frac{d^{2} \boldsymbol{a}_{2}}{a_{2}^{2 \gamma}}\left|\tilde{V}^{(\beta, \gamma)}\left(\boldsymbol{a}_{2}\right)\right|^{2} .
\end{aligned}
$$

Define $C_{\tilde{v}, \gamma}=\iint_{\mathbb{R}^{2}} \omega^{-2 \gamma}\left|\tilde{V}^{(\beta, \gamma)}(\boldsymbol{\omega})\right|^{2} d^{2} \boldsymbol{\omega}$, so that with $\tilde{C}_{o}^{-1}=C_{v, \gamma}, G(\cdot)$ is reconstructed. By direct calculation $C_{\bar{v}, \gamma}=8 \pi^{2} /(r-1)$, and recall $r>1$. $\mu_{g}\left(\mathcal{D}_{2}^{(\beta, \gamma)}(C)\right)=\left\langle\tilde{\mathcal{P}}_{\mathcal{A}_{2}(C) ; \beta, \gamma} g, \tilde{\mathcal{P}}_{\mathcal{A}_{2}(C) ; \beta, \gamma} g\right\rangle /\langle g, g\rangle$ determines the localization of $g(\cdot) \in L^{2}\left(\mathbb{R}^{2}\right)$, to any given $\mathcal{D}_{2}^{(\beta, \gamma)}(C)$.

Furthermore when $g(\boldsymbol{x})=g_{I}(\boldsymbol{x})$, the operator is related to $\mathcal{P}_{\mathcal{A}^{+}(\mathcal{C}) ; \beta, \gamma}$. In the frequency domain, from (9), with radial $g_{I}(\boldsymbol{x}), \mathcal{A}_{2}=\mathcal{A}_{2}^{+}(C)$ and $\tilde{v}_{\boldsymbol{\xi}}^{(\beta, \gamma)}(\boldsymbol{x})=\bar{v}_{\boldsymbol{\xi}}^{(\beta, \gamma)}(\boldsymbol{x})$ we obtain

$$
\begin{aligned}
& \tilde{\mathcal{P}}_{\mathcal{A}_{2}^{+}(C) ; \beta, \gamma}\left\{G_{I}\right\}\left(\frac{\boldsymbol{\omega}_{1}}{2 \pi}\right) \\
& \quad=\tilde{C}_{O} \int_{\mathcal{A}_{2}^{+}(C)} \bar{V}_{\boldsymbol{\xi}}^{(\beta, \gamma)}\left(\omega_{1}\right)\left\langle\bar{v}_{\boldsymbol{\xi}}^{(\beta, \gamma)}, g_{I}\right\rangle d A_{\boldsymbol{\xi}} \\
& \quad=\overline{\mathcal{P}}_{\mathcal{A}_{2}^{+}(C) ; \beta, \gamma} G_{I}\left(\frac{\boldsymbol{\omega}_{1}}{2 \pi}\right) \\
& \quad=\bar{C}_{O} \int_{\omega_{2}>0} \iint_{a^{2}+b^{2}+1 \leq 2 a C} \kappa\left(\omega_{1}, \omega_{2} ; a, b\right) \frac{d a}{a^{2}} d b d \omega_{2} .
\end{aligned}
$$

Note that $V^{(1-D, \beta, \gamma)}(\omega) \approx 0$ if $\omega$ is sufficiently small and the term $J_{0}\left(\omega_{l}^{\gamma} b\right)$ for $\omega_{l}^{\gamma} b \gg 0$, is considered as the point $b=0$ has zero measure in the plane. For $|z| \gg 0$ the asymptotic approximation to the zeroth Bessel function is $J_{0}(z) \approx$ $(\sqrt{2} / \sqrt{\pi z}) \cos (z-(\pi / 4))$, cf. [24]. We require $|\arg z|<\pi$ however, this is not an issue as $b>0$ and $\omega_{l}>0$. For fixed nonzero $b$ for values such that the integrand is nonzero

$$
\begin{aligned}
\kappa(\boldsymbol{\omega} ; a, b) & \\
= & 2 \pi a^{1 / \gamma}\left(\omega_{1} \omega_{2}\right)^{(-1+\gamma) / 2} V^{(1-D, \beta, \gamma)}\left(a^{1 / \gamma} \omega_{1}\right) \\
& \times \omega_{2} V^{(1-D, \beta, \gamma) *}\left(a^{1 / \gamma} \omega_{2}\right) G_{I}\left(\frac{\omega_{2}}{2 \pi}\right) J_{0}\left(b \omega_{1}^{\gamma}\right) J_{0}\left(b \omega_{2}^{\gamma}\right) b
\end{aligned}
$$

$\kappa(\boldsymbol{\omega} ; a, b)$

$$
\begin{aligned}
\approx & 2 \pi \omega_{1}^{-1 / 2+\gamma / 2} V^{(1-D, \beta, \gamma)}\left(a^{1 / \gamma} \omega_{1}\right) \omega_{2}^{1 / 2+\gamma / 2} \\
& \times a^{1 / \gamma} V^{(1-D, \beta, \gamma) *}\left(a^{1 / \gamma} \omega_{2}\right) G_{I}\left(\frac{\omega_{2}}{2 \pi}\right) b \sqrt{\frac{2}{\pi b \omega_{2}^{\gamma}}} \\
& \times \cos \left(\omega_{2}^{\gamma} b-\frac{\pi}{4}\right) \sqrt{\frac{2}{\pi b \omega_{1}^{\gamma}}} \cos \left(\omega_{1}^{\gamma} b-\frac{\pi}{4}\right) \\
= & 2 a^{1 / \gamma} V^{(1-D, \beta, \gamma)}\left(a^{1 / \gamma} \omega_{1}\right) \sqrt{\frac{\omega_{2}}{\omega_{1}}} G_{I}\left(\frac{\omega_{2}}{2 \pi}\right) \\
& \times V^{(1-D, \beta, \gamma) *}\left(a^{1 / \gamma} \omega_{2}\right) \cos \left(\left(\omega_{1}^{\gamma}-\omega_{2}^{\gamma}\right) b\right) \\
& +2 a^{1 / \gamma} V^{(1-D, \beta, \gamma)}\left(a^{1 / \gamma} \omega_{1}\right) \sqrt{\frac{\omega_{2}}{\omega_{1}}} G_{I}\left(\frac{\omega_{2}}{2 \pi}\right) \\
& \times V^{(1-D, \beta, \gamma) *}\left(a^{1 / \gamma}\left(\omega_{2}\right)\right) \cos \left(\left(\omega_{1}^{\gamma}+\omega_{2}^{\gamma}\right) b-\frac{\pi}{2}\right) .
\end{aligned}
$$


The integration is over $\omega_{2}>0$ and also $\omega_{1}>0$, hence, the first term of (23) dominates over the second term. The integrand of $\kappa\left(\omega_{1}, \omega_{2} ; a, b\right)$ can be replaced by $\kappa_{2}\left(\omega_{1}, \omega_{2} ; a, b\right)$ defined in (13) of the previous section. The operator when acting on $G_{I}(\cdot)$ in the frequency domain is then approximated via

$$
\begin{aligned}
\tilde{\mathcal{P}}_{\mathcal{A}_{2}^{+}(C) ; \beta, \gamma}\left\{G_{I}\right\}\left(\boldsymbol{\omega}_{1}\right) \approx & \bar{C}_{o} \int_{\omega_{2}=0}^{\infty} \iint_{\mathcal{A}^{+} C} \frac{d a}{a^{2}} d b d \omega_{2} \\
& \kappa_{2}\left(\omega_{1}, \omega_{2} ; a, b\right) \\
\equiv & \mathcal{P}_{\mathcal{A}^{+}(C) ; \beta, \gamma}\left\{G_{I}\right\}\left(\frac{\boldsymbol{\omega}_{1}}{2 \pi}\right) .
\end{aligned}
$$

Defining $\tilde{\mathcal{P}}_{\mathcal{A}_{2}(C) ; \beta, \gamma}$ using $v_{\boldsymbol{\xi}}(\boldsymbol{x})=\tilde{v}_{\boldsymbol{\xi}}^{(\beta, \gamma)}(\boldsymbol{x})$ and specifying $\mathcal{A}_{2}=\mathcal{A}_{2}(C)$ in (7) allows us to calculate the localization of an arbitrary $L^{2}\left(\mathbb{R}^{2}\right)$ function to $\mathcal{D}_{2}^{(\beta, \gamma)}(C)$. The radial eigenfunctions of $\tilde{\mathcal{P}}_{\mathcal{A}_{2}^{+}(C) ; \beta, \gamma}$ are approximately those of $\mathcal{P}_{\mathcal{A}^{+}(C) ; \beta, \gamma}$, where the derivation of the approximation shows the reasoning behind the definition of $\mathcal{P}_{\mathcal{A}^{+}(C) ; \beta, \gamma}$ in more detail. Finally, $\tilde{\mathcal{P}}_{\mathcal{A}_{2} ; \beta, \gamma}$ can be generalized to an arbitrary localization by removing the constraint of radial symmetry in $\mathcal{A}_{2}$, and using nonradial $\tilde{v}_{\boldsymbol{\xi}}^{(\beta, \gamma)}(\boldsymbol{x})$.

\section{E. Eigenfunctions: The Isotropic Wavelet}

From (19), note that the isotropic 2-D Morse wavelets are the eigenfunctions of $\mathcal{P}_{\mathcal{A}^{+}(C) ; \beta, \gamma}$, given for $l=\beta-1 / 2, \gamma=m$, $r=(2 \beta+1) / \gamma$, and fixed $n \in \mathbb{N}$ by

$$
\begin{aligned}
\Psi_{n ; l, m}^{(e)}(\boldsymbol{f})= & \Psi_{n ; l, m}^{(e)}(f)=\sqrt{2} A_{n ; l+1 / 2, m}(2 \pi f)^{l} e^{-(2 \pi f)^{m}} \\
& L_{n}^{c_{l, m}^{\prime}}\left(2(2 \pi f)^{m}\right)
\end{aligned}
$$

in terms of $f=|f|$. Equation (24) is (19) with the 1-D Morse wavelets $\Psi_{n ; \beta, \gamma}^{(1-D, e)}(f)$ substituted into the equation from [3]. The quantity $A_{n ; \beta, \gamma}=\sqrt{\pi \gamma 2^{r} \Gamma(n+1) / \Gamma(n+r)}$ is a normalization factor, $c_{l, m}^{\prime}=(2 l+2) / m-1$, and $L_{n}^{c}(\cdot)$ is a generalized Laguerre polynomial [23, p. 783]. The spatial domain wavelets with $x=|\boldsymbol{x}|$ are determined by the inverse FT of (24). Since they are isotropic this is equivalent to $\psi_{n}^{(e)}(\boldsymbol{x})=2 \pi \int_{0}^{\infty} \Psi^{(e)}(f) J_{0}(2 \pi f x) f d f$, where $J_{0}(x)$ is the zeroth Bessel function. Fig. 1 shows the $(l, m)=(8,3)$ isotropic 2-D Morse wavelets in the spatial domain [(a) $n=0$, and (b) $n=1]$ and their modulus in the spatial frequency domain [(c) $n=0$, and (d) $n=1]$. Their radially symmetric oscillatory structure is very clear, and their spatial decay is radial from the spatial origin. Their modulus in the spatial frequency domain demonstrates that they are bandpass filters, nonzero for a range of frequencies centred at the same distance from the spatial frequency origin. The trough in the modulus of the $n=1$ wavelet follows from the orthogonality of $\Psi_{0 ; l, m}^{(e)}(f)$ and $\Psi_{1 ; ; l, m}^{(e)}(f)$. The sum of the moduli squared, i.e., $\left|\Psi_{0 ; l, m}^{(e)}(f)\right|^{2}+\left|\Psi_{1 ; l, m}^{(e)}(f)\right|^{2}$, will be large in a ring-shape
structure.

To characterize the frequency content of the $n$th wavelet, suppressing the $(l, m)$ dependence, define

$$
f_{\max }^{(n)}=\arg _{f>0} \max \left|\Psi_{n ; l, m}^{(e)}(f)\right|^{2}, \quad n=0, \ldots, N-1 .
$$

The magnitude square of the FT of analysis wavelet $\psi_{\boldsymbol{\xi}, n ; l, m}^{(e)}(\cdot)$ will have a maximum at frequency $f_{\max }^{(n)} / a$ and is unaffected by
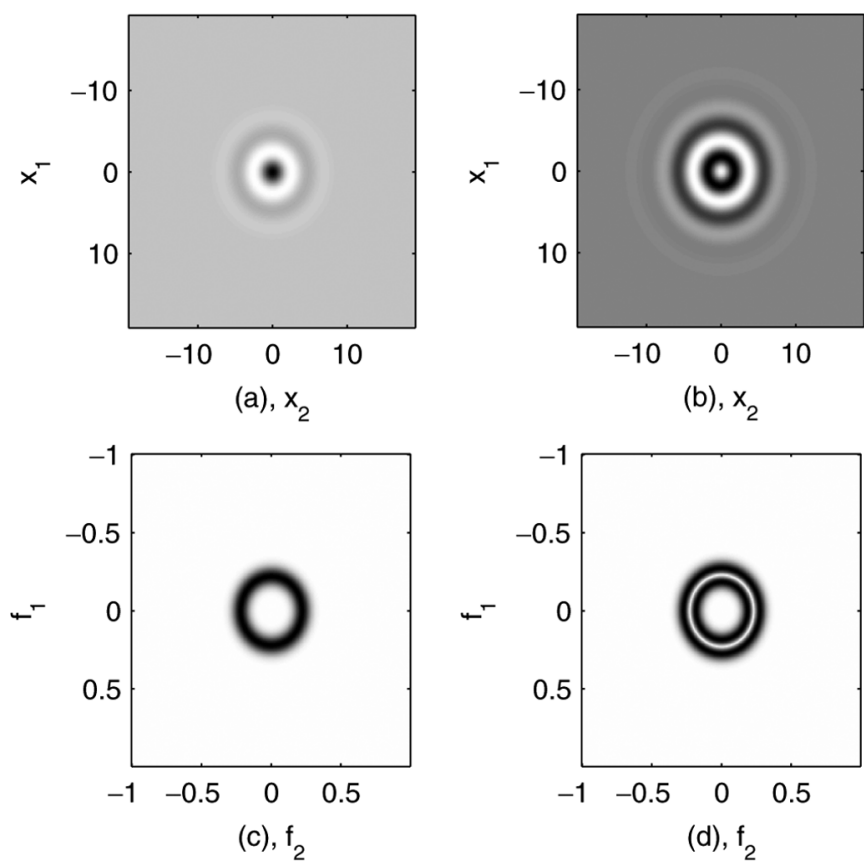

Fig. 1. The isotropic Morse wavelets in the spatial domain, $(l, m)=(8,3)$ and $n=0$ (a), $n=1$ (b). The modulus of the isotropic Morse wavelets in the spatial frequency domain, $(l, m)=(8,3)$ and $n=0$ (c), $n=1$ (d).

both $\boldsymbol{b}$ and the rotation. Recall that in the analysis, as the eigenvalues decrease, and thus the spatial localization of $\psi_{n ; l, m}^{(e)}(\cdot)$ deteriorate with $n$, only a finite number $n=0, \ldots, N-1$ will be used in direct analog to [1]. Analysis using the isotropic Morse mother wavelets cannot extract directional structure as $\psi_{n ; l, m}^{(e)}\left(\boldsymbol{r}_{-\theta} \boldsymbol{x}\right)=\psi_{n ; l, m}^{(e)}(\boldsymbol{x}), \forall n$.

\section{LOCAL PHASE DESCRIPTIONS OF VARIATION}

\section{A. The Riesz Transformations}

In 1-D, the analytic signal is used to unambiguously define the amplitude and phase of a given real signal. Using an analytic analysis wavelet defines the local magnitude and phase of a signal at each time and scale point via the WT. This is a necessity for the analysis of multicomponent signals [10]. A local oscillation in 1-D is defined by its amplitude and phase where the phase describes the dominant local instantaneous frequency of the signal, whereas the amplitude gives the local energy contribution. The analytic signal is constructed in the frequency domain by removing all negative frequencies in the signal, and then inverting the FT - and so any real signal $g(x)$ is complemented by its Hilbert transform $\mathcal{H}\{g\}(x)$. The analytic signal corresponds to $g^{+}(x)=g(x)+j \mathcal{H}\{g\}(x)$. If an oscillation is persistent over a range of times, then it will be heavily weighted in the Fourier domain, and the analytic signal approximately takes the form of a complex exponential.

The correct extension of the analytic signal to 2-D has been the subject of much debate — of particular note are perhaps the single orthant image of [25], the hypercomplex signal of [26] and the monogenic signal of [9]. To calculate the monogenic extension of a real image $g(\boldsymbol{x})$ its two Riesz transforms [9] are defined: $\mathcal{R}_{s} g(\boldsymbol{x}) \equiv g^{(s)}(\boldsymbol{x})=\left(r_{s} \circ g\right)(\boldsymbol{x})$, and $r_{s}(\boldsymbol{x})=$ 
$\left(x_{s} /(2 \pi) x^{3}\right), s=1,2$, where $\circ$ as before denotes the 2-D convolution. The FTs of $r_{s}(\boldsymbol{x})$ are given by

$$
R^{(1)}(\boldsymbol{f})=-\boldsymbol{j} \cos (\phi), \quad R^{(2)}(\boldsymbol{f})=-\boldsymbol{j} \sin (\phi) .
$$

The full Riesz transform of an image $g(\boldsymbol{x})$ is represented as $\mathcal{R} g(\boldsymbol{x})=\boldsymbol{i} \mathcal{R}_{1} g(\boldsymbol{x})+\boldsymbol{j} \mathcal{R}_{2} g(\boldsymbol{x})$. Define the monogenic image [9] as $g^{(+)}(\boldsymbol{x})=g(\boldsymbol{x})+\mathcal{R} g(\boldsymbol{x})$. This is a quaternionic object, and following [9] a local amplitude, orientation and phase of the image $g(\boldsymbol{x})$, may be defined from $g^{(+)}(\boldsymbol{x})$.

\section{B. Locally Oscillatory Signals}

Images that locally exhibit intrinsically 1-D oscillations will be considered, and such images appear, for instance, in machine vision, granular flow, and general oriented patterns [27]. They can be modeled by a sum of locally modulated sinusoids as follows:

$$
\begin{aligned}
c(\boldsymbol{x}) & =\sum_{l=1}^{L} c_{l}(\boldsymbol{x}) \\
c_{l}(\boldsymbol{x}) & =m_{l}(\boldsymbol{x}) \cos \left(2 \pi \phi_{l}(\boldsymbol{x})\right), \quad l=1, \ldots, L
\end{aligned}
$$

and let $\boldsymbol{n}_{l}\left(\boldsymbol{x}_{0}\right) \quad \overline{\bar{T}} \quad \nabla \phi_{l}\left(\boldsymbol{x}_{0}\right) /\left|\nabla \phi_{l}\left(\boldsymbol{x}_{0}\right)\right|=$ $\left[\cos \left(\eta_{l}\left(\boldsymbol{x}_{0}\right)\right) \sin \left(\eta_{l}\left(\boldsymbol{x}_{0}\right)\right)\right]^{T}$, and $\phi_{l}(\boldsymbol{x})=\varphi_{l}\left(\boldsymbol{n}_{l}(\boldsymbol{x}) \cdot \boldsymbol{x}\right)$. The unit vector $\boldsymbol{n}_{l}(\boldsymbol{x})$ is assumed to be varying slowly, in comparison with $\boldsymbol{x}$, across the spatial period, and the local variable $y_{l}(\boldsymbol{x})=\boldsymbol{n}_{l}(\boldsymbol{x}) \cdot \boldsymbol{x}$ is defined. $\boldsymbol{n}_{l}(\boldsymbol{x})$ corresponds to the orientation modulation (OM), whereas $\varphi_{l}(\cdot)$ is the phase modulation of component $1 . \varphi_{l}(\cdot)$ as a function of $y_{l}(\boldsymbol{x})$ plays the same role as the phase/frequency modulation (FM) of a 1-D signal. Assume $m_{l}(\boldsymbol{x})$ varies slowly in comparison to the cosine term, and corresponds to the amplitude modulation (AM). For examples of real images that can be modelled in such a form, see Fig. 4(a) (T03_03.jpg), as well as Fig. 4(b) (T24_17.jpg added to T24_23.jpg) from [30]. The former is a bark pattern, where analysis of the local structure would give information regarding its formation process, the latter is a combination of corduroy patterns, and is an example of a structure whose local characterization could be the basis of a segmentation algorithm of a picture containing multiple patterns. It follows for $\boldsymbol{x}=x_{0}+\boldsymbol{\delta} \boldsymbol{x}$, with $\varphi_{l}^{\prime}\left(y_{l}\right)=d / d y_{l}\left[\varphi_{l}\left(y_{l}\right)\right]$, and $\phi_{l}^{\prime}(\boldsymbol{x})=\varphi_{l}^{\prime}\left(\boldsymbol{n}_{l}(\boldsymbol{x}) \cdot \boldsymbol{x}\right)$, that $\phi_{l}(\boldsymbol{x})=\phi_{l}\left(\boldsymbol{x}_{0}\right)+\phi_{l}^{\prime}\left(\boldsymbol{x}_{0}\right) \boldsymbol{n}_{l}\left(\boldsymbol{x}_{0}\right) \cdot\left(\boldsymbol{x}-\boldsymbol{x}_{0}\right)+O\left(|\boldsymbol{\delta} \boldsymbol{x}|^{2}\right)$. The monogenic image of the lth component is, in terms of $\boldsymbol{e}_{\eta_{l}}\left(\boldsymbol{x}_{0}\right)=\boldsymbol{i} \cos \left(\eta_{l}\left(\boldsymbol{x}_{0}\right)\right)+\boldsymbol{j} \sin \left(\eta_{l}\left(\boldsymbol{x}_{0}\right)\right)$, given by

$$
\begin{aligned}
& c_{l}^{(+)}(\boldsymbol{x}) \\
& =m_{l}\left(\boldsymbol{x}_{0}\right) e^{2 \pi \boldsymbol{e}_{\eta_{l}}\left(\boldsymbol{x}_{0}\right)\left(\phi_{l}\left(\boldsymbol{x}_{0}\right)+\phi_{l}^{\prime}\left(\boldsymbol{x}_{0}\right) \boldsymbol{n}_{l}\left(\boldsymbol{x}_{0}\right) \cdot\left(\boldsymbol{x}-\boldsymbol{x}_{0}\right)\right)} \\
& \quad \times e^{2 \pi \boldsymbol{e}_{\eta_{l}}\left(\boldsymbol{x}_{0}\right)\left(O\left(\left|\boldsymbol{x}-\boldsymbol{x}_{0}\right|^{2}\right)\right)} \\
& =m_{l}\left(\boldsymbol{x}_{0}\right)\left[\begin{array}{c}
\cos \left(2 \pi\left\{\phi_{l}\left(\boldsymbol{x}_{0}\right)+\phi_{l}^{\prime}\left(\boldsymbol{x}_{0}\right) \boldsymbol{n}_{l}\left(\boldsymbol{x}_{0}\right) \cdot\left(\boldsymbol{x}-\boldsymbol{x}_{0}\right)\right\}\right) \\
+\boldsymbol{e}_{\eta_{l}}\left(\boldsymbol{x}_{0}\right) \sin \left(2 \pi \left\{\phi_{l}\left(\boldsymbol{x}_{0}\right)+\phi_{l}^{\prime}\left(\boldsymbol{x}_{0}\right) \boldsymbol{n}_{l}\left(\boldsymbol{x}_{0}\right)\right.\right. \\
\left.\left.\left.\cdot\left(\boldsymbol{x}-\boldsymbol{x}_{0}\right)\right\}\right)\right]+\tilde{r}\left(\boldsymbol{x} ; \boldsymbol{x}_{0}\right)
\end{array}\right.
\end{aligned}
$$

combining the calculation of the Riesz transformation of a sinusoid with linear phase [28], with a Taylor expansion of the oscillation, and defining the error term as $\tilde{r}\left(\boldsymbol{x} ; \boldsymbol{x}_{0}\right)$. Assuming the orientation $\boldsymbol{e}_{\eta_{l}}\left(\boldsymbol{x}_{0}\right)$ is constant across values of $\boldsymbol{x}$ for which $m_{l}(\boldsymbol{x})$ is nonzero, we shall calculate the FT of $c_{l}^{(+)}(\boldsymbol{x})$ in terms of the unit quaternion $\boldsymbol{e}_{\eta_{l}}\left(\boldsymbol{x}_{0}\right)$ instead of $\boldsymbol{j}$. De Moivre's theorem is still valid for any unit quaternion, and so $C_{\boldsymbol{e}_{l}, l}^{(+)}(\boldsymbol{f})$ can be interpreted in terms of a decomposition in terms of oscillations in direction $\eta_{l}\left(\boldsymbol{x}_{0}\right)$. Note that

$$
\begin{aligned}
C_{\boldsymbol{e}_{\eta_{l}}, l}^{(+)}(\boldsymbol{f})= & \iint_{\mathbb{R}^{2}} d^{2} \boldsymbol{x} m_{l}\left(\boldsymbol{x}_{0}\right) e^{2 \pi \boldsymbol{e}_{\eta_{l}}\left(\boldsymbol{x}_{0}\right) \phi_{l}\left(\boldsymbol{x}_{0}\right)} \\
& \times e^{2 \pi \boldsymbol{e}_{\eta_{l}}\left(\boldsymbol{x}_{0}\right)\left(\phi_{l}^{\prime}\left(\boldsymbol{x}_{0}\right) \boldsymbol{n}_{l}\left(\boldsymbol{x}_{0}\right) \cdot\left(\boldsymbol{x}-\boldsymbol{x}_{0}\right)-\boldsymbol{f} \cdot \boldsymbol{x}\right)} \\
& +\tilde{R} \boldsymbol{e}_{\eta_{l}}\left(\boldsymbol{f} ; \boldsymbol{x}_{0}\right) .
\end{aligned}
$$

We apply the stationary phase approximation to the above integral [29]. We assume that $\Omega(\boldsymbol{x}, \boldsymbol{f})=\phi_{l}\left(\boldsymbol{x}_{0}\right)+\phi_{l}^{\prime}\left(\boldsymbol{x}_{0}\right) \boldsymbol{n}_{l}\left(\boldsymbol{x}_{0}\right)$. $\left(\boldsymbol{x}-\boldsymbol{x}_{0}\right)-\boldsymbol{f} \cdot \boldsymbol{x}$ has the unique stationary point $\boldsymbol{x}_{0}$, and that there is quadratic behavior around this point, i.e., $\Omega(\boldsymbol{x}, \boldsymbol{f})=(1 / 2)\left[\left(\boldsymbol{x}-\boldsymbol{x}_{0}\right)^{T} \boldsymbol{H}\left(\boldsymbol{x}-\boldsymbol{x}_{0}\right)\right]$, where $\boldsymbol{H}$ is the Hessian matrix of $\Omega(\boldsymbol{x}, \boldsymbol{f})$. Under these assumptions we find that the only nonnegligible contribution in the integral of (29) is at $\boldsymbol{f}=\boldsymbol{f}\left(\boldsymbol{x}_{0}\right)=\phi_{l}^{\prime}\left(\boldsymbol{x}_{0}\right) \boldsymbol{n}_{l}\left(\boldsymbol{x}_{0}\right)$, where

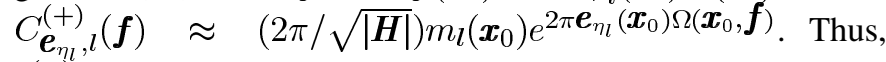
$c_{l}^{(+)}(\boldsymbol{x})$ provides a local description of component $l$ near $\boldsymbol{x}=\boldsymbol{x}_{0}$, in terms of a local oscillation with parameters $\left(m_{l}, \phi_{l}^{\prime}, \boldsymbol{n}_{l}\right)$, that may be determined from (28), just like the analytic signal allows for the determination of a magnitude and a phase in 1-D. The instantaneous frequency of the local oscillations is $\left|\phi_{l}^{\prime}\left(x_{0}\right)\right|$, where the orientation of these oscillations, is given by $\boldsymbol{n}_{l}\left(\boldsymbol{x}_{0}\right)$. The sign of $\phi_{l}^{\prime}\left(\boldsymbol{x}_{0}\right)$ is taken so that the orientation angle is restricted from $-(\pi / 2)$ to $(\pi / 2)$. Finally, the local magnitude $m_{l}\left(\boldsymbol{x}_{0}\right)$ has the interpretation of local energy presence.

If the image is actually a sum of several AM/FM/OM terms present at the same spatial location, i.e., the signal is multicomponent, as is the case in Fig. 4(b) and also often in many observed images, we will not be able to use $c^{(+)}(\boldsymbol{x})$ directly to determine the properties of the local oscillations. This is due to the $L$ components not being separated. The corresponding problem with multi-component signals in 1-D is much documented [10]. Generally, to analyze multicomponent signals localized methods are combined with the analytic signal construction and for this purpose the local monogenic descriptions of images will be calculated, cf. ridge analysis [29]. The local description will be able to resolve the individual components depending on the choice of wavelet function $\psi(\cdot)$. The descriptions may when different oscillations are present at the same spatial location extract well-behaved orientation, phase and amplitude functions locally, and for this reason monogenic wavelets are defined and used.

\section{Monogenic Wavelets}

In 1-D the analytic Morse wavelets can be constructed from the even Morse wavelets. The even Morse wavelet is invariant to sign changes of the argument, or time direction, whereas the Hilbert transform of the even wavelet corresponds to the odd 
Morse wavelet. A monogenic version of isotropic wavelets in 2-D is constructed, based on the real isotropic Morse wavelets, using the Riesz transforms. The monogenic wavelets are represented as quaternionic functions defined for each $n$ via

$$
\begin{aligned}
\psi_{n ; l, m}^{(+)}(\boldsymbol{x}) & =\psi_{n ; l, m}^{(e)}(\boldsymbol{x})+\boldsymbol{i} \psi_{n ; l, m}^{(1)}(\boldsymbol{x})+\boldsymbol{j} \psi_{n ; l, m}^{(2)}(\boldsymbol{x}) \\
& =\psi_{n ; l, m}^{(e)}(\boldsymbol{x})+\psi_{n ; l, m}^{(q)}(\boldsymbol{x})
\end{aligned}
$$

where the FT of the real part of the monogenic wavelet is given by (24) and the additional two real functions are defined in the Fourier domain as Riesz transforms of the isotropic wavelet function via

$$
\begin{aligned}
\Psi_{n, l, m}^{(s)}(\boldsymbol{f})= & -\boldsymbol{j} \sqrt{2} A_{n ; l+1 / 2, m} \frac{f_{s}}{f}(2 \pi f)^{l} e^{-(2 \pi f)^{m}} \\
& L_{n}^{c_{l, m}^{\prime}}\left(2(2 \pi f)^{m}\right), \quad s=1,2 .
\end{aligned}
$$

We consider $\psi_{n ; l, m}^{(q)}(\boldsymbol{x})=\boldsymbol{i} \psi_{n ; l, m}^{(1)}(\boldsymbol{x})+\boldsymbol{j} \psi_{n ; l, m}^{(2)}(\boldsymbol{x})$ as a single object. $\psi_{n ; l, m}^{(q)}(\boldsymbol{x})$ has the same norm as $\psi_{n ; l, m}^{(e)}(\boldsymbol{x})$. The entire monogenic wavelet thus has twice the norm of the isotropic real wavelet, i.e., $\left\|\psi_{n ; l, m}^{(+)}\right\|^{2}=2\left\|\psi_{n ; l, m}^{(e)}\right\|^{2}$. Fig. 2 shows $\psi_{n ; 8,3}^{(1)}(\boldsymbol{x})$ plotted in the spatial domain [(a) $n=0$ and (b) $n=1$ ], as well as the moduli of $\Psi_{n ; 8,3}^{(1)}(\boldsymbol{f})$ in the spatial frequency domain [(c) $n=0$ and (b) $n=1$ ]. Note that, although the real component of the monogenic wavelet is isotropic, the two Riesz components of the monogenic wavelet are odd in the $x_{1}$ and $x_{2}$ directions respectively. In the following, $(l, m)$ are fixed and their values suppressed for notational convenience. We define here the translated, rotated and dilated wavelet as $\psi_{n, \boldsymbol{\xi}}^{(+)}(\boldsymbol{x})=\mathcal{R}_{\theta} \mathcal{D}_{a} \mathcal{T}_{\boldsymbol{b}} \psi_{n}^{(+)}(\boldsymbol{x})$. This can also be thought of as the sum of translating, rotating, and dilating each of the real components of the mother wavelet. The continuous WT of an image $g(\cdot)$ with respect to either the components of, or with respect to the full quaternionic wavelet, is defined as $w_{n}^{(\cdot)}(\boldsymbol{\xi} ; g)=\int d^{2} \boldsymbol{x} g(\boldsymbol{x}) \psi_{n, \boldsymbol{\xi}}^{(\cdot) *}(\boldsymbol{x})$. The associated scalogram is given by $S_{n}^{(\cdot)}(\boldsymbol{\xi} ; g)=\left|w_{n}^{(\cdot)}(\boldsymbol{\xi} ; g)\right|^{2}$. The WT of image $g(\cdot)$ is then given either in the spatial domain, or spatial frequency domain, in terms of $\boldsymbol{\zeta}=\left[a, \theta, \boldsymbol{f}_{\boldsymbol{b}}\right]^{T}$, where $\boldsymbol{f}_{\boldsymbol{b}}$ is the Fourier variable for $\boldsymbol{b}$ via

$$
\begin{aligned}
w_{n}^{(+)}(\boldsymbol{\xi} ; g) & =w_{n}^{(e)}(\boldsymbol{\xi} ; g)-\boldsymbol{i} w_{n}^{(1)}(\boldsymbol{\xi} ; g)-\boldsymbol{j} w_{n}^{(2)}(\boldsymbol{\xi} ; g) \\
W_{n}^{(+)}(\boldsymbol{\zeta} ; g) & =\left[1-\boldsymbol{k} \cos \left(\phi_{b}-\theta\right)+\sin \left(\phi_{b}-\theta\right)\right] \\
W_{n}^{(e)}(\boldsymbol{\zeta} ; g) & =a \Psi_{n}^{(+)}\left(a \boldsymbol{r}_{-\theta} \boldsymbol{f}_{b}\right) G\left(\boldsymbol{f}_{b}\right) .
\end{aligned}
$$

These equations follow by direct calculation.

The WT can, thus, be understood in terms of the FT of $\psi_{n, \boldsymbol{\xi}}^{(+)}(\boldsymbol{x})$, given by $\Psi_{n, \boldsymbol{\xi}}^{(+)}(\boldsymbol{f})=$ $[1-\boldsymbol{k} \cos (\phi-\theta)+\sin (\phi-\theta)] \Psi_{n, \boldsymbol{\xi}}^{(e)}(\boldsymbol{f}) . \quad \Psi_{n, \boldsymbol{\xi}}^{(+)}(\boldsymbol{f}) \quad$ has modulus $\left|\Psi_{n, \boldsymbol{\xi}}^{(+)}(\boldsymbol{f})\right|^{2}=2(1+\sin (\phi-\theta))\left|\Psi_{n, \boldsymbol{\xi}}^{(e)}(\boldsymbol{f})\right|^{2}$, and the modulus of the real isotropic wavelet is invariant with respect to $\theta$. In terms of $a$, the monogenic wavelet is band passing the image to frequencies with period $a^{-1} f_{\max }^{(n)}$, whereas the term $\sin (\phi-\theta)$ is repositioning the wavelet in orientation depending on the value of $\theta$.

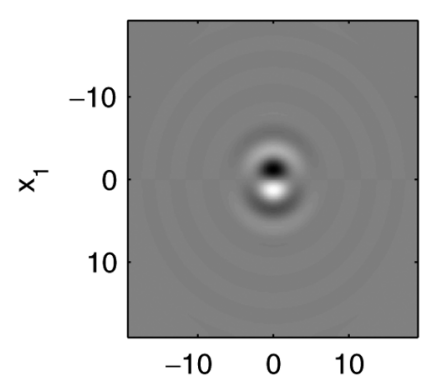

(a), $x_{2}$

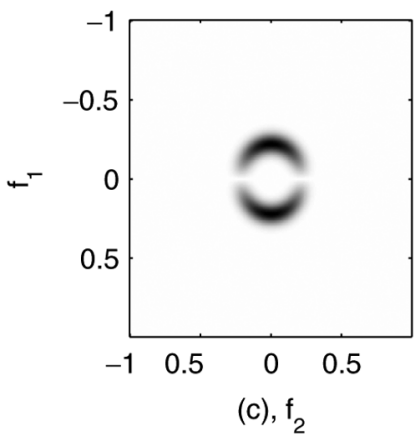

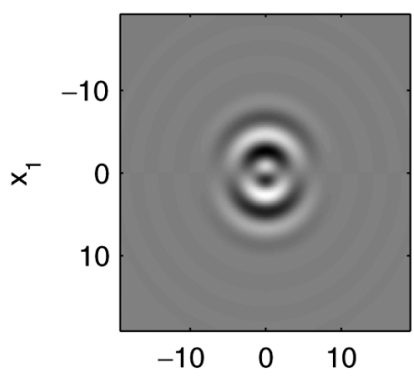

(b), $x_{2}$

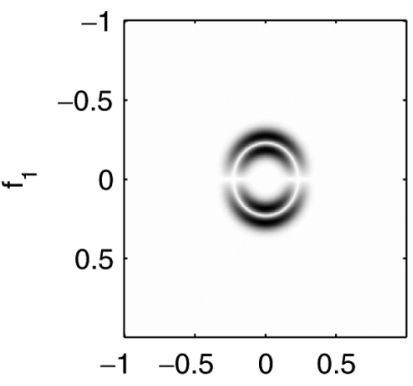

(d), $f_{2}$
Fig. 2. For $(l, m)=(8,3)$ : the $x_{1}$ Riesz transform Morse wavelets in the spatial domain for $n=0$ (a), and $n=1$ (b). The modulus of the $x_{1}$ Riesz transform Morse wavelets in the spatial frequency domain for $n=0$ (c), and $n=1$ (d).

When using more than one mother wavelet, letting $n=0,1,2, \ldots$, we may note the following orthogonality relations between the wavelets

$$
\begin{aligned}
& \left\langle\psi_{n_{1}}^{(e)}, \psi_{n_{2}}^{(e)}\right\rangle=\delta_{n_{1}, n_{2}}, \quad\left\langle\psi_{n_{1}}^{(s)}, \psi_{n_{2}}^{(s)}\right\rangle=\frac{1}{2} \delta_{n_{1}, n_{2}} \\
& \left\langle\psi_{n_{1}}^{(1)}, \psi_{n_{2}}^{(2)}\right\rangle=0, \quad\left\langle\psi_{n_{1}}^{(e)}, \psi_{n_{2}}^{(s)}\right\rangle=0, \quad s=1,2 .
\end{aligned}
$$

The multiple Morse wavelets thus combine to form an orthogonal system, and this will have implications for their usage when performing estimation of local characteristics of real images. The total energy of the image using the $n$th wavelet only is given by

$$
\begin{aligned}
S_{n}^{(+)}(\boldsymbol{\xi} ; g) & =S_{n}^{(e)}(\boldsymbol{\xi} ; g)+S_{n}^{(1)}(\boldsymbol{\xi} ; g)+S_{n}^{(2)}(\boldsymbol{\xi} ; g) \\
& =S_{n}^{(e)}(\boldsymbol{\xi} ; g)+S_{n}^{(q)}(\boldsymbol{\xi} ; g) .
\end{aligned}
$$

For any fixed $n=0,1,2, \ldots, N-1$, recast the full WT of the noise as a vector with real valued entries, $\boldsymbol{w}_{n}(\boldsymbol{\xi} ; g)$ $=\left[\begin{array}{lll}w_{n}^{(e)}(\boldsymbol{\xi} ; g) & w_{n}^{(1)}(\boldsymbol{\xi} ; g) \quad w_{n}^{(2)}(\boldsymbol{\xi} ; g)\end{array}\right]^{T}$. Then we note that as $\psi_{n}^{(e)}(\cdot)$ is radially symmetric with $\tilde{\boldsymbol{r}}_{\theta}=\left[\begin{array}{lll}1 & 0 & 0\end{array}\right.$ $\left.\left[\begin{array}{ll}0 & 0\end{array}\right]^{T} \boldsymbol{r}_{\theta}\right]$

$$
\boldsymbol{w}_{n}(\boldsymbol{\xi} ; g)=\tilde{\boldsymbol{r}}_{-\theta} \boldsymbol{w}_{n}\left(\boldsymbol{\xi}_{0} ; g\right)
$$

where $\boldsymbol{\xi}_{0}=[a, 0, \boldsymbol{b}]$. The WT only needs to be calculated at one orientation, unlike the case with directional wavelets that filter the wavelets selectively in the frequency domain. The prevalent local directional structure of the signal at that position and scale may be determined from the three wavelet coefficients at each $\xi_{0}$. 


\section{The Monogenic WT}

The WT separates out the signal into local behavior, so that different components present in the same signal can be estimated. The Sections V-A and B relate typical local structures in terms of their characterizations via their wavelet coefficients.

\section{A. The Monogenic WT of Discontinuities}

The idealized version of a point discontinuity at $x_{0}$ corresponds to $g_{s, 1}(\boldsymbol{x})=m_{1}(\boldsymbol{x}) \delta\left(\boldsymbol{x}-\boldsymbol{x}_{0}\right)$, where $m_{1}(\boldsymbol{x})$ is assumed to be a well-behaved function at point $\boldsymbol{x}=\boldsymbol{x}_{0}$. This singularity is characterized by $\boldsymbol{x}_{0}$, its location, and $m_{1}\left(\boldsymbol{x}_{0}\right)$, the amplitude at the location. The WT of this object is $\left.w^{(+)}(\boldsymbol{\xi} ; g)=m_{1}\left(\boldsymbol{x}_{0}\right) \psi_{\boldsymbol{\xi}}^{(+) *}\left(\boldsymbol{r}_{-\theta}\left(\boldsymbol{x}_{0}-\boldsymbol{b}\right) / a\right)\right) / a$. This is maximum near $\boldsymbol{b}=\boldsymbol{x}_{0}$ where the function has a modulus square of $m_{1}^{2}\left(\boldsymbol{x}_{0}\right)\left|\psi^{(+)}(\mathbf{0})\right|^{2} / a^{2}$. Thus, point singularities can be located by finding maxima in $\boldsymbol{b}$. Furthermore $\left|w^{(+)}(\boldsymbol{\xi} ; g)\right|^{2}$ has no dependence on $\theta$, and as the magnitude of the wavelet at the origin is known, $m_{1}(\cdot)$ can be determined.

A 1-D singularity is modeled as $g_{s, 2}(\boldsymbol{x})=m_{2}\left(x_{1}\right)$ $\delta\left(\cos \left(\theta_{2}\right) x_{1}+\sin \left(\theta_{2}\right) x_{2}-c\right)$. The line $x_{2}=c\left(\theta_{2}=\pi / 2\right)$ modulated by the value of $m_{2}\left(x_{1}\right)$ is permitted, however, we do not permit the line $x_{1}=c\left(\theta_{2}=0\right)$, as this would lead to an image of infinite energy. Also, we constrain $-(\pi / 4)<\theta_{2} \leq(3 \pi / 4)$, for calculational convenience. Assume that $m_{2}\left(x_{1}\right)$ is a symmetric function around a maximum at $x_{1}=x_{1, \max }$. We characterize the structure of $g_{s, 2}(\cdot)$, using the WT. The WT with the isotropic mother wavelet, noting that for some $x_{s} \in \mathbb{R}^{+}, \psi_{n}^{(e)}(x) \approx 0 \forall x>x_{s}$, is given by the equation shown at the bottom of the page). $\left|w_{n}^{(e)}\left(\boldsymbol{\xi}, g_{s, 2}\right)\right|$ will be large for values of $\boldsymbol{b}$ such that

$\sqrt{\left(x_{1, \max }-b_{1}\right)^{2}+\left(c \csc \left(\theta_{2}\right)-\cot \left(\theta_{2}\right) x_{1, \max }-b_{2}\right)^{2}}<x_{s} / a$.

Similar results hold for $w_{n}^{(l)}\left(\boldsymbol{\xi}, g_{s, 2}\right)$, as $\psi_{n}^{(l)}(x), l=1,2$ roughly have the same spatial support as $\psi_{n}^{(e)}(x)$. The location of the singularity for any fixed value $a$ is identified as $\boldsymbol{b}=\left(x_{1, \max }, c \csc \left(\theta_{2}\right)-\cot \left(\theta_{2}\right) x_{1, \max }\right)$, as maxima in the modulus of the WT using the monogenic wavelet. The orientation $\theta_{2}$ will be visually apparent from the $\boldsymbol{b}$ plane and can be determined at a fixed point $\boldsymbol{\xi}$. The FT of $g_{s, 2}(\boldsymbol{x})$ is

$$
\begin{aligned}
G_{s, 2}(\boldsymbol{f})= & \frac{M_{2}\left(f_{1}-\cot \left(\theta_{2}\right) f_{2}\right)}{\left|\sin \left(\theta_{2}\right)\right|} \\
& \times e^{-\boldsymbol{j} 2 \pi\left(f_{1} \cos \left(\theta_{2}\right)+f_{2} \sin \left(\theta_{2}\right)\right) c} \\
& \times e^{-\boldsymbol{j} 2 \pi\left(-\sin \left(\theta_{2}\right) f_{1}+\cos \left(\theta_{2}\right) f_{2}\right) c \cot \left(\theta_{2}\right)}
\end{aligned}
$$

where $M_{2}(\cdot)$ is the FT of $m_{2}(\cdot)$. The WTs of the Riesz components of this image are

$$
W_{n}^{(l)}\left(\boldsymbol{\zeta}_{0} ; g_{s, 2}\right)=\boldsymbol{j}\left(f_{b, l} / f_{b}\right) a \Psi_{n}^{(e)}\left(a f_{b}\right) G_{s, 2}\left(\boldsymbol{f}_{b}\right), \quad l=1,2
$$

where $\zeta_{0}=\left[a, 0, \boldsymbol{f}_{\boldsymbol{b}}\right]$. The FT of the rotated wavelet is by (35) given by

$$
\begin{aligned}
& W_{n}^{(1)}\left(\boldsymbol{\zeta} ; g_{s, 2}\right)= \\
& \quad \boldsymbol{j}\left(\left(\cos (\theta) f_{b, 1}+\sin (\theta) f_{b, 2}\right) / f_{b}\right) a \Psi_{n}^{(e)}\left(a f_{b}\right) G_{s, 2}(\boldsymbol{f})
\end{aligned}
$$

with $W_{n}^{(2)}\left(\zeta ; g_{s, 2}\right)$ following mutatis mutandis. Calculating the wavelet transform at $\theta=\theta_{2}$, with $\boldsymbol{\xi}_{2}=\left[a, \theta_{2}, \boldsymbol{b}\right]$

$$
\begin{aligned}
w_{n}^{(1)}\left(\boldsymbol{\xi}_{2} ; g_{s, 2}\right)= & \frac{a \boldsymbol{j}}{|\sin (\theta)|} \int_{-\infty}^{\infty} \int_{-\infty}^{\infty} d f_{\theta, b, 2} d f_{\theta, b, 1} \frac{f_{\theta, b, 1}}{f_{\theta, b}} \\
& \times e^{\boldsymbol{j} 2 \pi f_{\theta, b, 1}\left[\left(\boldsymbol{r}_{-\theta} \boldsymbol{b}\right)_{1}-c\right]} \\
& \times \Psi_{n}^{(e)}\left(a f_{\theta, b}\right) M_{2}\left(-\frac{f_{\theta, b, 2}}{\sin (\theta)}\right) \\
& \times e^{\boldsymbol{j} 2 \pi\left[f_{\theta, b, 2}\left(\boldsymbol{r}_{-\theta} \boldsymbol{b}\right)_{2}-f_{\theta, b, 2} c \cot (\theta)\right]} \\
= & \frac{1}{|\sin (\theta)|} \int_{-\infty}^{\infty} d f_{\theta, b, 1} \boldsymbol{j} \frac{f_{\theta, b, 1}}{f_{\theta, b}} \\
& \times e^{\boldsymbol{j} 2 \pi f_{\theta, b, 1}\left[\left(\boldsymbol{r}_{-\theta} \boldsymbol{b}\right)_{1}-c\right]} \\
& \times \tilde{M}_{2}\left(f_{\theta, b, 1}^{2} ; \boldsymbol{\xi}_{2}, c\right) .
\end{aligned}
$$

Equation (36) defines $\tilde{M}_{2}\left(f_{\theta, b, 1}^{2} ; \boldsymbol{\xi}_{2}, c\right)$, as an even function of $f_{\theta, b, 1}$, and $\boldsymbol{f}_{\theta, b}=\left[f_{\theta, b, 1}, f_{\theta, b, 2}\right]=\boldsymbol{r}_{-\theta} \boldsymbol{f}_{b}$. For fixed values of $\left(a, \theta_{2}\right)$ a value of $\boldsymbol{b}$ such that $\left(\boldsymbol{r}_{-\theta} \boldsymbol{b}\right)_{1}=c$, denoted $\boldsymbol{b}_{2}$, can be found. When $\boldsymbol{\xi}=\boldsymbol{\xi}_{2}^{\prime}=\left[a, \theta_{2}, \boldsymbol{b}_{2}\right]$ then $\sin \left\{\left(2 \pi f_{\theta, b, 1}\right)\left[\left(\boldsymbol{r}_{-\theta} \boldsymbol{b}\right)_{1}-c\right]\right\} \quad$ vanishes identically for all $f_{\theta, b, 1}$. Hence, $w_{n}^{(1)}\left(\boldsymbol{\xi}_{2}^{\prime} ; g_{s, 2}\right)=0$, while from (35) it follows that the energy of the WT with $\psi^{(q)}(\cdot)$ is conserved under rotations. Thus $\left|w^{(2)}(\boldsymbol{\xi} ; g)\right|$ is maximum at $\theta=\theta_{2}$, and $\boldsymbol{b}=\boldsymbol{b}_{2}$. With the correct choice of rotation $\theta$ the angle $\theta_{2}$ can be found by maximizing the energy of $w_{n}^{(2)}\left(\boldsymbol{\xi} ; g_{s, 2}\right)$ and minimizing the energy of $w_{n}^{(1)}\left(\boldsymbol{\xi} ; g_{s, 2}\right)$. If the magnitudes of the two WTs at $\boldsymbol{\xi}=\boldsymbol{\xi}_{0}$ are equal then $\theta=3 \pi / 4$, otherwise take a value of $\theta$ that maximises $\varrho(\theta)=w_{n}^{(2) 2}\left(\boldsymbol{\xi}_{0} ; g_{s, 2}\right)-w_{n}^{(1) 2}\left(\boldsymbol{\xi}_{0} ; g_{s, 2}\right) . \varrho(\theta)$ has a stationary point at

$$
\theta_{\max , n}=\frac{1}{2} \tan ^{-1}\left(\frac{2 w_{n}^{(1)}\left(\boldsymbol{\xi}_{0} ; g_{s, 2}\right) w_{n}^{(2)}\left(\boldsymbol{\xi}_{0} ; g_{s, 2}\right)}{w_{n}^{(1) 2}\left(\boldsymbol{\xi}_{0} ; g_{s, 2}\right)-w_{n}^{(2) 2}\left(\boldsymbol{\xi}_{0} ; g_{s, 2}\right)}\right)
$$

corresponding to a maximum if the appropriate solution is chosen. Note that if $\left|w_{n}^{(2)}\left(\boldsymbol{\xi}_{0} ; g_{s, 2}\right)\right|>\left|w_{n}^{(1)}\left(\boldsymbol{\xi}_{0} ; g_{s, 2}\right)\right|$ with $-(\pi / 4)<\theta<(\pi / 4), \theta_{n, \max }$ is a maximum whereas if $\left|w_{n}^{(2)}\left(\boldsymbol{\xi}_{0} ; g_{s, 2}\right)\right|<\left|w_{n}^{(1)}\left(\boldsymbol{\xi}_{0} ; g_{s, 2}\right)\right|$ the restriction $(\pi / 4)<\theta \leq(\pi / 2)$, or $-(\pi / 2)<\theta<-(\pi / 4)$ is made. Thus at any fixed point $\boldsymbol{\xi}_{0}$ the orientation that would result from a line-discontinuity passing through $\boldsymbol{b}$ can be determined by utilizing the above equation, and this characterizes local orientational structure. A line discontinuity can be locally characterized, once separated from the rest of the signal using the

$$
w_{n}^{(e)}\left(\boldsymbol{\xi}, g_{s, 2}\right)=\int_{-\infty}^{\infty} d x_{1} m_{2}\left(x_{1}\right) a^{-1} \psi_{n}^{(e)}\left(a^{-1} \sqrt{\left(x_{1}-b_{1}\right)^{2}+\left(c \csc \left(\theta_{2}\right)-\cot \left(\theta_{2}\right) x_{1}-b_{2}\right)^{2}}\right)
$$


monogenic Morse wavelet transform, in terms of its wavelet coefficients by its strength, given by maxima in $\left|w_{n}^{(+)}\left(\boldsymbol{\xi} ; g_{s, 2}\right)\right|^{2}$, and its local direction, namely $\theta_{\max }$.

\section{B. The Monogenic WT of AM/FM/OM Images}

Analysis of AM/FM/OM images of the form given by (27), is considered in this subsection. Then

$$
\begin{aligned}
w_{n}^{(e)}\left(\boldsymbol{\xi} ; c_{p}\right)= & \frac{1}{2} \int_{\mathbb{R}^{2}} m_{p}(\boldsymbol{x})\left[e^{2 \boldsymbol{j} \pi \phi_{p}(\boldsymbol{x})}+e^{-2 \boldsymbol{j} \pi \phi_{p}(\boldsymbol{x})}\right] \\
& \psi_{\boldsymbol{\xi}, n}^{(e)}(\boldsymbol{x}) d^{2} \boldsymbol{x} \\
= & \frac{m_{p}(\boldsymbol{b})}{2} \int_{\mathbb{R}^{2}}\left[e^{2 \boldsymbol{j} \pi\left(\phi_{p}(\boldsymbol{b})+\phi_{p}^{\prime}(\boldsymbol{b}) \boldsymbol{n}_{p}(\boldsymbol{b}) \cdot(\boldsymbol{x}-\boldsymbol{b})\right)}\right. \\
& \left.\quad+e^{-2 \boldsymbol{j} \pi\left(\phi_{p}(\boldsymbol{b})+\phi_{p}^{\prime}(\boldsymbol{b}) \boldsymbol{n}_{p}(\boldsymbol{b}) \cdot(\boldsymbol{x}-\boldsymbol{b})\right)}\right] \\
& \cdot \psi_{\boldsymbol{\xi}, n}^{(e)}(\boldsymbol{x}) d^{2} \boldsymbol{x}+o(1) \\
= & m_{p}(\boldsymbol{b}) \cos \left[2 \pi \phi_{p}(\boldsymbol{b})\right] a \Psi_{n}^{(e)}\left(a \phi_{p}^{\prime}(\boldsymbol{b})\right)+o(1)
\end{aligned}
$$

where $p \in\{1, \ldots, L\}$. Also

$$
\begin{aligned}
w_{n}^{(u)}\left(\boldsymbol{\xi}_{0} ; c_{p}\right)= & \frac{m_{p}(\boldsymbol{b})}{2} \int_{\mathbb{R}^{2}}\left[e^{2 \boldsymbol{j} \pi\left(\phi_{p}(\boldsymbol{b})+\phi_{p}^{\prime}(\boldsymbol{b}) \boldsymbol{n}_{p}(\boldsymbol{b})(\boldsymbol{x}-\boldsymbol{b})\right)}\right) \\
\left.+e^{-2 \boldsymbol{j} \pi\left(\phi_{p}(\boldsymbol{b})+\phi_{p}^{\prime}(\boldsymbol{b}) \boldsymbol{n}_{p}(\boldsymbol{b})(\boldsymbol{x}-\boldsymbol{b})\right)}\right] & \\
& \cdot \psi_{\boldsymbol{\xi}, n}^{(u) *}(\boldsymbol{x}) d^{2} \boldsymbol{x}+o(1) \\
= & \frac{1}{2} m_{p}(\boldsymbol{b}) a\left[e^{2 \boldsymbol{j} \pi \phi_{p}(\boldsymbol{b})} \Psi_{n}^{(u) *}\left(a \phi_{p}^{\prime}(\boldsymbol{b}) \boldsymbol{n}_{p}(\boldsymbol{b})\right)+\right. \\
& \left.e^{-2 \boldsymbol{j} \pi \phi_{p}(\boldsymbol{b})} \Psi_{n}^{(u) *}\left(-a \phi_{p}^{\prime}(\boldsymbol{b}) \boldsymbol{n}_{p}(\boldsymbol{b})\right)\right]+o(1),
\end{aligned}
$$

$u=1$, 2. It follows from (35) that $w_{n}^{(+)}\left(\boldsymbol{\xi} ; c_{p}\right)=$ $m_{p}(\boldsymbol{b}) a \Psi_{n}^{(e)}\left(a \phi_{p}^{\prime}(\boldsymbol{b})\right) e^{2 \pi \boldsymbol{e}_{\nu_{p}} \phi_{p}(\boldsymbol{b})}+o(1)$, with $\boldsymbol{e}_{\nu_{p}}=$ $\boldsymbol{i} \cos \left(\eta_{p}(\boldsymbol{b})-\theta\right)+\boldsymbol{j} \sin \left(\eta_{p}(\boldsymbol{b})-\theta\right), \nu_{p}=\eta_{p}(\boldsymbol{b})-\theta$, corresponding to the local analog of (28). For multicomponent images, by a suitable choice of wavelets we may be able to separate the components, in the spirit of ridge analysis based on complex wavelets [29]. Ridge analysis requires the assumption $m_{p_{1}}(\boldsymbol{b}) a \Psi_{n}^{(e)}\left(a \phi_{p_{1}}^{\prime}(\boldsymbol{b})\right) \gg m_{p_{2}}(\boldsymbol{b}) a \Psi_{n}^{(e)}\left(a \phi_{p_{2}}^{\prime}(\boldsymbol{b})\right) \forall p_{1} \neq p_{2}$, at all $\boldsymbol{\xi}$ considered, which imposes a constraint on $\Psi_{n}^{(e)}(f)$, and corresponds to a choice of $(n, l, m)$.

Furthermore, the modulus of the WT is $\left|w^{(+)}\left(\boldsymbol{\xi} ; c_{p}\right)\right|^{2}=$ $m_{p}^{2}(\boldsymbol{b}) a^{2} \Psi_{n}^{(e) 2}\left(a \phi_{p}^{\prime}(\boldsymbol{b})\right)$, and the WT of $c_{p}(\cdot)$ is locally maximal on the curve given by $\mathcal{R}(a, \theta, \boldsymbol{b})=\left\{(a, \boldsymbol{b}): a \phi_{p}^{\prime}(\boldsymbol{b})=f_{\max }^{(n)}\right\}$, where $f_{\max }^{(n)}$ is given by (25). This defines the monogenic wavelet ridges [29] of an AM/FM/OM image, where at any point on this ridge, the local orientation may be computed. Ridge analysis is based on the fact that not all information of the redundant WT representation needs to be used to characterize the image. As the ridge definition does not depend on the angle $\theta$, the transform can be calculated at only one value of $\theta$. The oscillatory components are characterized by their local orientation, amplitude, and oscillation frequency

$$
\begin{aligned}
\nu_{p}(\boldsymbol{b})= & \tan ^{-1}\left(\frac{w^{(2)}\left(\boldsymbol{\xi} ; c_{p}\right)}{w^{(1)}\left(\boldsymbol{\xi} ; c_{p}\right)}\right)=\eta_{p}(\boldsymbol{b})-\theta \\
a_{p}^{2}(\boldsymbol{b})= & \frac{\left|w^{(+)}\left(\boldsymbol{\xi} ; c_{p}\right)\right|^{2}}{a^{2} \Psi^{(e) 2}\left(a \phi_{p}^{\prime}(\boldsymbol{b})\right)} \\
\phi_{p}(\boldsymbol{b})= & \frac{1}{2 \pi} \tan ^{-1}\left(\frac{\sqrt{w^{(1) 2}\left(\boldsymbol{\xi} ; c_{p}\right)+w^{(2) 2}\left(\boldsymbol{\xi} ; c_{p}\right)}}{w^{(e)}\left(\boldsymbol{\xi} ; c_{p}\right)}\right) \\
& \times \operatorname{sgn}\left(w^{(2)}\left(\boldsymbol{\xi} ; c_{p}\right)\right) .
\end{aligned}
$$

We have constrained $-(\pi / 2) \leq \nu_{p} \leq(\pi / 2)$ and $-(1 / 2) \leq$ $\phi_{p} \leq(1 / 2)$ by the choice of sign for the $\tan ^{-1}(\cdot)$. Hence, having isolated a local oscillatory component at point $\boldsymbol{\xi}$, it can be characterized by (38). For further discussion of the analysis of AM/FM/OM signals using the monogenic WT, including examples of univariate as well as bivariate analyses, see Metikas and Olhede [31], [32].

\section{DigitAl IMPLEMENTATION AND ESTIMATION}

\section{A. Digital Implementation}

Given sampled image $\left\{g\left(s_{1} \Delta_{1}, s_{2} \Delta_{2}\right)\right\}_{s_{1}=1, s_{2}=1}^{N_{1}, N_{2}}$, where the sampling period is $\Delta_{1}$ and $\Delta_{2}$ in $x_{1}$ and $x_{2}$ respectively, to preserve the exact monogenic structure the WT is implemented from the Fourier domain. The implementation follows very much in the spirit of Olhede and Walden [3], and is calculated by the inverse discrete FT, thus, making the algorithm of order $O\left(N_{1} N_{2} \log \left(N_{1}\right) \log \left(N_{2}\right)\right)$. With $N_{u}^{\prime}=\left[N_{u} / 2\right]$, and $[x]$ denoting integer part, it follows:

$$
\begin{aligned}
& w_{n}^{(s)}\left(a, 0, b_{1} \Delta_{1}, b_{2} \Delta_{2}\right) \\
&=\int_{-\left(1 / 2 \Delta_{1}\right)}^{\left(1 / 2 \Delta_{1}\right)} \int_{-\left(1 / 2 \Delta_{2}\right)}^{\left(1 / 2 \Delta_{2}\right)} R^{(s) *}(\boldsymbol{f}) G(\boldsymbol{f}) \\
& \times a \Psi_{n}^{(e)}(a f) e^{2 \boldsymbol{j} \pi \boldsymbol{f} \cdot \boldsymbol{b}} d^{2} \boldsymbol{f} \\
&= \frac{a}{N_{1} N_{2} \Delta_{1} \Delta_{2}} \sum_{l_{1}=-N_{1}^{\prime}}^{N_{1}^{\prime}-1} \sum_{l_{2}=-N_{2}^{\prime}}^{N_{2}^{\prime}-1} R^{(s) *}\left(\frac{l_{1}}{N_{1} \Delta_{1}}, \frac{l_{2}}{N_{2} \Delta_{2}}\right) \\
& \times G\left(\frac{l_{1}}{N_{1} \Delta_{1}}, \frac{l_{2}}{N_{2} \Delta_{2}}\right) \Psi_{n}^{(e)}\left(a \sqrt{\frac{l_{1}^{2}}{N_{1}^{2} \Delta_{1}^{2}}+\frac{l_{2}^{2}}{N_{2}^{2} \Delta_{2}^{2}}}\right) \\
& \times e^{2 j \pi\left(\left(l_{1} / N_{1}\right) b_{1}+\left(l_{2} / N_{2}\right) b_{2}\right)}+O\left(\frac{1}{\min \left(N_{1}, N_{2}\right)}\right)
\end{aligned}
$$

where $s=e, 1,2, b_{1}=0, \ldots, N_{1}-1, b_{2}=0, \ldots, N_{2}-1$, and $n=0, \ldots, N . R^{(e)}(\boldsymbol{f}) \equiv 1$, and $R^{(s)}(\boldsymbol{f}), s=1,2$ are given by (26). The WT at any value of $\theta$ can be found from (35) and by calculating each individual $w_{n}^{(s)}\left(a, 0, b_{1} \Delta_{1}, b_{2} \Delta_{2}\right), s=e, 1$, 2 from (39). For a discretely sampled image the WT can then be calculated easily by the above procedure for $\theta \in(0,2 \pi)$ and $\boldsymbol{b} \in\left(0, N_{1} \Delta_{1}\right) \times\left(0, N_{2} \Delta_{2}\right)$. Also note the maximum and minimum scales that can be resolved: the real 2-D even wavelet 
$\Psi_{n}^{(e)}(\cdot)$ is built from a real 1-D wavelet corresponding to an essentially supported bandpass filter, and, thus, there exists frequencies $f_{1}^{(n)}$ and $f_{2}^{(n)}$ [3], such that

$$
\Psi_{n}^{(e)}(f) \approx 0 \quad \forall f:|f| \notin\left(f_{1}^{(n)}, f_{2}^{(n)}\right) .
$$

Assume that sampling is sufficiently fine so that $G(\boldsymbol{f})=0$ for all frequencies not in the Nyquist band. The WT can be calculated only at scales $a$ such that $\Psi_{n}^{(e)}\left(a \sqrt{f_{1}^{2}+f_{2}^{2}}\right) \approx$ $0 \forall \boldsymbol{f}:\left|f_{1}\right| \geq 1 /\left(2 \Delta_{1}\right),\left|f_{2}\right| \geq 1 /\left(2 \Delta_{2}\right)$. This necessitates $a \geq a_{\min }=2 f_{2}^{(n)}\left(\Delta_{1} \Delta_{2} / \sqrt{\Delta_{1}^{2}+\Delta_{2}^{2}}\right)$. As $a$ increases in magnitude, the wavelet becomes more peaked in the frequency domain, and to ensure the wavelet covers at least $M$ frequency points we constrain $a \leq a_{\max }=$ $(1 / M) \min \left(N_{1} \Delta_{1}, N_{2} \Delta_{2}\right)\left[f_{2}^{(n)}-f_{1}^{(n)}\right]$. This gives clear description on how to calculate the WT, and for what ranges of the parameters the transform coefficients are meaningful.

\section{B. Statistical Properties}

Consider estimation of features present in an image immersed in white noise where the image is collected in a regular grid consisting of $x_{l}=s_{l} \Delta_{l}, s_{l}=0, \ldots, N_{l}-1$, $l=1$, 2. The observed image $y_{s_{1}, s_{2}}$ is modelled as $y_{s_{1}, s_{2}}=g\left(x_{1}, x_{2}\right)+\epsilon_{s_{1}, s_{2}}$, with $\epsilon_{s_{1}, s_{2}}$ assumed to be isotropically Gaussian and white. It is assumed that $E\left(\epsilon_{s_{1}, s_{2}}\right)=0$, and $E\left(\epsilon_{s_{1}, s_{2}}, \epsilon_{u_{1}, u_{2}}\right)=\delta_{s_{1}, u_{1}} \delta_{s_{2}, u_{2}} \sigma_{\epsilon}^{2}$. The WT of the noise will also be Gaussian, as it corresponds to a sum of jointly Gaussian variables. Thus to determine the distribution of the wavelet coefficients, its first and second order structure is found at a fixed $\boldsymbol{\xi}$. The WT is a linear operation and $w_{n}^{(\cdot)}(\boldsymbol{\xi} ; y)=w_{n}^{(\cdot)}(\boldsymbol{\xi} ; g)+w_{n}^{(\cdot)}(\boldsymbol{\xi} ; \epsilon)$. It follows that $E\left(w_{n}^{(\cdot)}(\boldsymbol{\xi} ; y)\right)=w_{n}^{(\cdot)}(\boldsymbol{\xi} ; g)$, and the second order structure of the estimators can be determined from the distribution of the noise coefficients. In the appendix, with the additional assumption of $\min \left(\left(1 /\left(2 \Delta_{1}\right)\right),\left(1 /\left(2 \Delta_{2}\right)\right)\right)>$ $\max _{n=0, \ldots, N-1} f_{\max }^{(n)}$, for fixed $n, \boldsymbol{w}_{n}(\boldsymbol{\xi} ; \epsilon) \stackrel{d}{=} \mathcal{N}_{3}\left(\mathbf{0}_{3}, \sigma_{\epsilon}^{2} \boldsymbol{V}\right)$, and $\boldsymbol{V}=\operatorname{diag}(1,1 / 2,1 / 2)$, where the latter denotes a diagonal matrix.

Any estimator of local signal properties needs to be smoothed, or averaged to obtain a low variance [1]. The WT using any of the specified wavelet functions averages the data across a window in space and spatial frequency, $\mathcal{D}^{(+; \beta, \gamma)}(C)$, apparent from Fig. 1(a)-(d) as the spatial and spatial frequency region the wavelets are essentially supported over. Thomson [1] suggested forming estimates of local properties by averaging local energy estimates using several orthogonal wavelets/functions. This usage explicitly reduces the variability of the estimates with a clearly specified averaging region $-\mathcal{D}^{(+; \beta, \gamma)}(C)$. Deterministic structure over $\mathcal{D}^{(+; \beta, \gamma)}(C)$ is reenforced across wavelet estimates, but the noisy uncorrelated behavior cancels. The bias inherent in the averaging is characterized by the eigenvalues square of the localization operator. In the Appendix, it is shown that $E\left(w_{n_{1}}^{\left(l_{1}\right)}(\boldsymbol{\xi} ; \epsilon) w_{n_{2}}^{\left(l_{2}\right)}(\boldsymbol{\xi} ; \epsilon)\right)=\sigma_{\epsilon}^{2} V_{l_{1} l_{2}} \delta_{n_{1}, n_{2}}$, and, thus, $\boldsymbol{w}_{n_{1}}(\boldsymbol{\xi} ; \epsilon)$ is uncorrelated (and independent from the Gaussianity assumptions on the errors) with $\boldsymbol{w}_{n_{2}}(\boldsymbol{\xi} ; \epsilon)$ unless $n_{1}=n_{2}$. Averages of the WTs and the scalograms across $n$ will be defined and used as a basis for calculating estimators of other quantities as $\bar{w}^{(l)}(\boldsymbol{\xi} ; \cdot)=N^{-1} \sum_{n=0}^{N-1} w_{n}^{(l)}(\boldsymbol{\xi} ; \cdot), \bar{S}^{(l)}(\boldsymbol{\xi} ; \cdot)=$ $N^{-1} \sum_{n=0}^{N-1} S_{n}^{(l)}(\xi ; \cdot)$, with $l=e, 1,2,+$. For $l_{1}, l_{2}=$ $e, 1,2,+$, and images $g_{1}(\cdot), g_{2}(\cdot), \bar{C}^{\left(l_{1}, l_{2}\right)}\left(\boldsymbol{\xi} ; g_{1}(\cdot), g_{2}(\cdot)\right)=$ $(1 / N) \sum_{n=0}^{N-1} w_{n}^{\left(l_{1}\right)}\left(\boldsymbol{\xi} ; g_{1}\right) w_{n}^{\left(l_{2}\right) *}\left(\boldsymbol{\xi} ; g_{2}\right), \quad$ is also defined. We define the estimators $\widehat{w}^{(l)}(\boldsymbol{\xi} ; g)=\bar{w}^{(l)}(\boldsymbol{\xi} ; y)$ and $\widehat{S}^{(l)}(\boldsymbol{\xi} ; g)=\bar{S}^{(l)}(\boldsymbol{\xi} ; y)$, for $l=e, 1,2,+$, as well as $w^{\left(l_{1}\right) w^{\left(l_{2}\right)}}\left(\boldsymbol{\xi} ; g_{1}\right)=\bar{C}^{\left(l_{1}, l_{2}\right)}\left(\boldsymbol{\xi} ; g_{1}(\cdot), g_{1}(\cdot)\right)$.

The Gaussian assumptions on $\epsilon$ then give $\overline{\boldsymbol{w}}(\boldsymbol{\xi} ; \epsilon)=$ $\left[\bar{w}^{(e)}(\boldsymbol{\xi} ; \epsilon), \bar{w}^{(1)}(\boldsymbol{\xi} ; \epsilon), \bar{w}^{(2)}(\boldsymbol{\xi} ; \epsilon)\right] \stackrel{d}{=} \mathcal{N}\left(\boldsymbol{O}_{\mathbf{3}}, \bar{\sigma}_{\epsilon}^{2} \boldsymbol{V}\right)$, where $\bar{\sigma}_{\epsilon}^{2}=\sigma_{\epsilon}^{2} / N$. For most quantities intuitively a reduction of $1 / N$ in the variances is achieved by the averaging. The energy of the image at point $\boldsymbol{\xi}$ is estimated by

$$
\begin{array}{r}
\widehat{S}^{(+)}(\boldsymbol{\xi} ; g)=\frac{1}{N} \sum_{n=0}^{N-1} S_{n}^{(+)}(\boldsymbol{\xi} ; y)=\bar{S}^{(+)}(\boldsymbol{\xi} ; g)+\bar{S}^{(+)}(\boldsymbol{\xi} ; \epsilon) \\
+\frac{2}{N} \sum_{n=0}^{N-1}\left[w_{n}^{(e)}(\boldsymbol{\xi} ; g) w_{n}^{(e)}(\boldsymbol{\xi} ; \epsilon)+w_{n}^{(1)}(\boldsymbol{\xi} ; g)\right. \\
\left.\cdot w_{n}^{(1)}(\boldsymbol{\xi} ; \epsilon)+w_{n}^{(2)}(\boldsymbol{\xi} ; g) w_{n}^{(2)}(\boldsymbol{\xi} ; \epsilon)\right] .
\end{array}
$$

Up to order $\bar{\sigma}_{\epsilon}^{2}$, with the additional assumption of the localized behavior of $g(\cdot)$ constant across the support of the $n=$ $0, \ldots, N-1$ wavelets, it can be shown

$$
\begin{aligned}
\widehat{S}^{(+)}(\boldsymbol{\xi} ; g) \stackrel{d}{=} \mathcal{N}\left(\bar{S}^{(+)}(\boldsymbol{\xi} ; g), 4 \frac{\sigma_{\epsilon}^{2}}{N}\left[\bar{S}^{(e)}(\boldsymbol{\xi} ; g)\right.\right. \\
\left.\left.+\frac{1}{2}\left(\bar{S}^{(1)}(\boldsymbol{\xi} ; g)+\bar{S}^{(2)}(\boldsymbol{\xi} ; g)\right)\right]\right)
\end{aligned}
$$

Hence the variance of the energy estimate decreases $O(1 / N)$.

\section{Distribution of Estimators}

We may estimate the orientation of a discontinuity potentially present at $\boldsymbol{\xi}_{0}=[a, 0, \boldsymbol{b}]$ by maximizing the difference between the energy of the second and first components. Each wavelet indexed by $n$ satisfies (37) and thus averaging over the equations gives

$$
\begin{aligned}
\theta_{2} & =\frac{1}{2} \tan ^{-1}\left(\frac{\left.2 \bar{C}^{(1,2)}\left(\boldsymbol{\xi}_{0} ; g_{s, 2}, g_{s, 2}\right)\right)}{\bar{S}^{(1)}\left(\boldsymbol{\xi}_{0} ; g_{s, 2}\right)-\bar{S}^{(2)}\left(\boldsymbol{\xi}_{0} ; g_{s, 2}\right)}\right) \\
\widehat{\theta}_{\max } & =\frac{1}{2} \tan ^{-1}\left(\frac{2 w^{\left(l_{1}\right) w^{\left(l_{2}\right)}}\left(\boldsymbol{\xi}_{0} ; g_{s, 2}\right)}{\widehat{S}^{(1)}\left(\boldsymbol{\xi}_{0} ; g_{s, 2}\right)-\widehat{S}^{(2)}\left(\boldsymbol{\xi}_{0} ; g_{s, 2}\right)}\right) .
\end{aligned}
$$

Let $w_{n}^{(l)}(\boldsymbol{\xi} ; \epsilon)=\sigma_{\epsilon} w_{n, \epsilon}^{(l)}$, which entails that $\bar{w}^{(l)}(\boldsymbol{\xi} ; \epsilon)=\sigma_{\epsilon} \bar{w}_{\epsilon}^{(l)}$ for $l=e, 1,2,+$, and expand the above expression $\widehat{\theta}_{\text {max }}=\theta_{2}+\sigma_{\epsilon} \delta \theta_{2}+O\left(\sigma_{\epsilon}^{2}\right)$. Note that $E\left(\delta \theta_{2}\right)=0$ : the estimator up to order $\sigma_{\epsilon}^{2}$ is, thus, unbiased, and with $\bar{P}^{(1,2)}\left(g_{s, 2}\right)=$ 
$\left[\bar{S}^{(1)}\left(g_{s, 2}\right)-\bar{S}^{(2)}\left(g_{s, 2}\right)\right]^{2}+4\left[\bar{C}^{(1,2)}\left(g_{s, 2}, g_{s, 2}\right)\right]^{2}$, the estimator has variance

$$
\operatorname{Var}\left[\widehat{\theta}_{\max }\right]=\frac{\sigma_{\epsilon}^{2}}{N} \frac{1}{2} \frac{\bar{S}^{(1)}\left(g_{s, 2}\right)+\bar{S}^{(2)}\left(g_{s, 2}\right)}{\bar{P}^{(1,2)}\left(g_{s, 2}\right)}+O\left(\sigma_{\epsilon}^{3}\right)
$$

Thus, using multiple wavelets leads to a variance reduction. For $\mathrm{AM} / \mathrm{FM} / \mathrm{OM}$ signals, we define the estimator for the orientation angle of the unit quaternion at any fixed local point $\boldsymbol{\xi}$ as

$$
\begin{aligned}
\widehat{\nu}_{l}\left(\boldsymbol{\xi} ; c_{l}\right)= & \bar{\nu}_{l}\left(\boldsymbol{\xi} ; y_{l}\right)=\tan ^{-1}\left[\frac{\widehat{w}^{(2)}\left(\boldsymbol{\xi} ; c_{l}\right)}{\widehat{w}^{(1)}\left(\boldsymbol{\xi} ; c_{l}\right)}\right] \\
= & \nu_{l}\left(\boldsymbol{\xi} ; c_{l}\right)+\sigma_{\epsilon} \frac{\bar{w}^{(1)}\left(\boldsymbol{\xi} ; c_{l}\right) \bar{w}_{\epsilon}^{(2)}-\bar{w}^{(2)}\left(\boldsymbol{\xi} ; c_{l}\right) \bar{w}_{\epsilon}^{(1)}}{\bar{S}^{(1)}\left(\boldsymbol{\xi} ; c_{l}\right)+\bar{S}^{(2)}\left(\boldsymbol{\xi} ; c_{l}\right)} \\
& +O\left(\sigma_{\epsilon}^{2}\right)
\end{aligned}
$$

Given the WT of the noise has expectation zero, the estimator is unbiased and it has variance, up to order $O\left(\sigma_{\epsilon}^{3}\right)$

$$
\begin{aligned}
\operatorname{Var}\left[\widehat{\nu}_{l}\left(\boldsymbol{\xi} ; c_{l}\right)\right] & =\frac{\sigma_{\epsilon}^{2}}{2 N} \frac{\bar{w}^{(1) 2}\left(\boldsymbol{\xi} ; c_{l}\right)+\bar{w}^{(2) 2}\left(\boldsymbol{\xi} ; c_{l}\right)}{\left[\bar{S}^{(1)}\left(\boldsymbol{\xi} ; c_{l}\right)+\bar{S}^{(2)}\left(\boldsymbol{\xi} ; c_{l}\right)\right]^{2}} \\
& =\frac{\sigma_{\epsilon}^{2}}{2 N^{2}} \frac{\left(\sum \Psi_{n_{1}}^{(e)}\left(a\left|\nabla \phi_{l}(\boldsymbol{b})\right|\right)\right)^{2}}{\sum \Psi_{n_{1}}^{(e) 2}\left(a\left|\nabla \phi_{l}(\boldsymbol{b})\right|\right)} \approx \frac{\sigma_{\epsilon}^{2}}{2 N} .
\end{aligned}
$$

Using multiple wavelets leads to variance reduction. To estimate the phase only a single wavelet is used, namely the $n=0$. Due to the orthogonality relations, the wavelet filters in the Fourier domain cannot be strictly positive for all frequencies, and, thus, for $n>0$ there are induced variations in the phase estimate whenever the wavelet filter changes sign. Such variations would give substantial bias in the phase estimate. Then: see (47) at the bottom of the page. As the expected value of the WT of noise is zero, the estimator is thus unbiased, and the variance of the phase estimator is

$$
\begin{aligned}
\operatorname{Var}\left[\widehat{\phi}_{l}\left(\boldsymbol{\xi} ; c_{l}\right)\right] & =\frac{\sigma_{\epsilon}^{2}}{S_{0}^{(+)}\left(\boldsymbol{\xi} ; c_{l}\right)} \frac{S_{0}^{(+)}\left(\boldsymbol{\xi} ; c_{l}\right)-\frac{1}{2} S_{0}^{(e)}\left(\boldsymbol{\xi} ; c_{l}\right)}{(2 \pi)^{2} S_{0}^{(+)}\left(\boldsymbol{\xi} ; c_{l}\right)} \\
& =\frac{\sigma_{\epsilon}^{2}\left[1-\frac{1}{2} \cos ^{2}\left(2 \pi \phi_{l}(\boldsymbol{b})\right)\right]}{(2 \pi)^{2} S_{0}^{(+)}\left(\boldsymbol{\xi} ; c_{l}\right)}
\end{aligned}
$$

When considering larger scales, the wavelets are averaging across many sample points, and the variance of the phase estimate decreases. The amplitude is estimated as $\widehat{a}_{l}^{2}(\boldsymbol{b})=\widehat{S}^{(+)}\left(\boldsymbol{\xi} ; c_{l}\right) /\left(a^{2} \bar{\Psi}^{(e) 2}\left(a \widehat{\phi}_{l}^{\prime}(\boldsymbol{b})\right)\right)$, $\bar{\Psi}^{(e) 2}(\cdot)=\sum_{n} \Psi_{n}^{(e) 2}(\cdot) / N$.

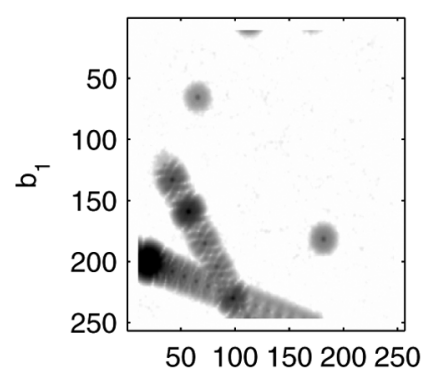

(a), $b_{2}$

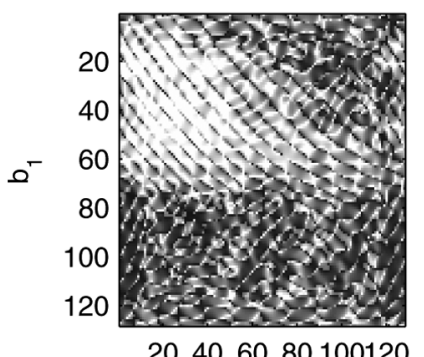

(c), $b_{2}$

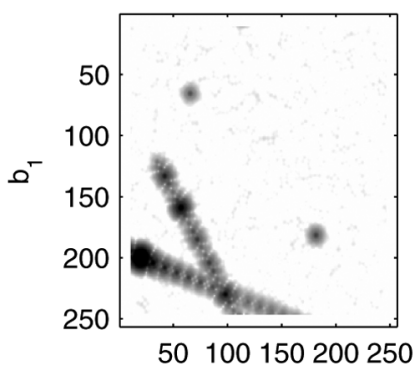

(b), $b_{2}$

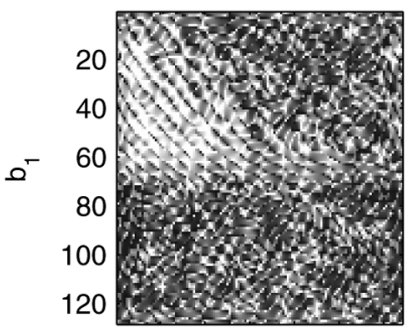

20406080100120

$(d), b_{2}$
Fig. 3. The local energy of signal 1 on a $\mathrm{dB}$ scale using three wavelets (a), or one wavelet (b), $a=1.4$, and $l=8, m=3$. The contour axis in (a) and (b) ranges from -7.5 (white) to 37 (black). The deviation of the estimated orientation of signal 2 from the true orientation at scale $a=1.66$ using dB scale, using three wavelets (c) and one wavelet (d). The contour axis in (c) and (d) ranges from -10 (white) to 5 (black).

\section{Examples}

To illustrate the wavelets' capacity of characterising local properties, and verify the theoretical variance reduction results we give some examples. Consider a collection of singularities observed in noise: $g_{1}(\boldsymbol{x})=\sum g_{1 j}(\boldsymbol{x})+\sigma_{1} \epsilon \boldsymbol{x}$, where

$g_{11}(\boldsymbol{x})=\left(10 /\left|\boldsymbol{x}-\left[N_{1} / 4+1 / 2 \quad N_{2} / 4+1 / 2\right]^{T}\right|\right)$

$g_{12}(\boldsymbol{x})=\left(15 /\left|\boldsymbol{x}-\left[\begin{array}{ll}45 N_{1} / 64+1 / 2 & 45 N_{2} / 64+1 / 2\end{array}\right]^{T}\right|\right)$

$g_{13}(x)=\left|x_{1} \cos (\pi / 3)-x_{2} \sin (\pi / 3)-(15 / 128) N_{1}+(1 / 2)\right|^{-1}$

$g_{14}(\boldsymbol{x})=\left|x_{1} \cos (\pi / 9)-x_{2} \sin (\pi / 9)-(45 / 64) N_{1}+(1 / 2)\right|^{-1}$

and we take $\operatorname{Var}\left(\epsilon_{\boldsymbol{x}}\right)=1$ as well as $\sigma_{1}=0.2$. The $g_{11}(\boldsymbol{x})$ and $g_{12}(\boldsymbol{x})$ are point singularities whereas $g_{13}(\boldsymbol{x})$ and $g_{14}(\boldsymbol{x})$ are line singularities. See Fig. 3(a) and (b) for a plot of the scalogram of the observed image at scale $a=1.4$, corresponding to radial frequencies of 0.17 . The discontinuities are clearly identified. The estimate using three wavelets of the local energy is a great deal more robust to the noise.

$$
\begin{aligned}
\widehat{\phi}_{l}\left(\boldsymbol{\xi} ; c_{l}\right)= & \phi_{0, l}\left(\boldsymbol{\xi} ; c_{l}\right)+O\left(\sigma_{\epsilon}^{2}\right)+\frac{\sigma_{\epsilon}}{2 \pi} \\
& \times\left[\frac{-\left(S_{0}^{(1)}\left(\boldsymbol{\xi} ; c_{l}\right)+S_{0}^{(2)}\left(\boldsymbol{\xi} ; c_{l}\right)\right) w_{\epsilon, 0}^{(e)}}{S_{0}^{(+)}\left(\boldsymbol{\xi} ; c_{l}\right) \sqrt{S_{0}^{(1)}\left(\boldsymbol{\xi} ; c_{l}\right)+S_{0}^{(2)}\left(\boldsymbol{\xi} ; c_{l}\right)}}+\frac{w_{0}^{(e)}\left(\boldsymbol{\xi} ; c_{l}\right)\left[w_{\epsilon, 0}^{(1)} w_{0}^{(1)}\left(\boldsymbol{\xi} ; c_{l}\right)+w_{\epsilon, 0}^{(2)} w_{0}^{(2)}\left(\boldsymbol{\xi} ; c_{l}\right)\right]}{S_{0}^{(+)}\left(\boldsymbol{\xi} ; c_{l}\right) \sqrt{S_{0}^{(1)}\left(\boldsymbol{\xi} ; c_{l}\right)+S_{0}^{(2)}\left(\boldsymbol{\xi} ; c_{l}\right)}}\right] .
\end{aligned}
$$




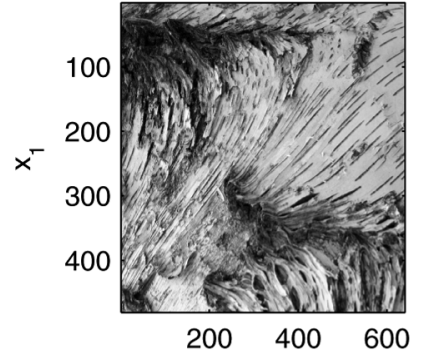

(a), $x_{2}$

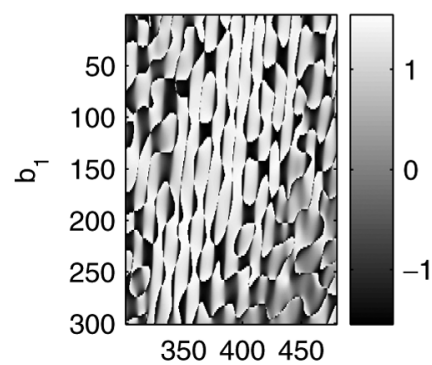

(c), $b_{2}$

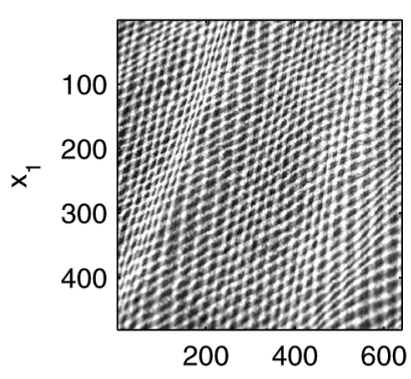

(b), $x_{2}$

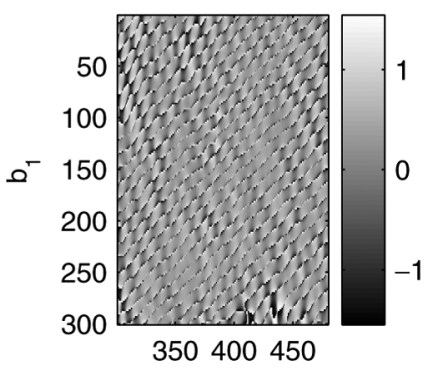

(d), $b_{2}$
Fig. 4. Two signals with typical AM/FM/OM structure (a) and (b). Local analysis of the image in (b) at positions $b_{1}=0, \ldots, 300$ and $b_{2}=301, \ldots, 480$ and scales $a=6.4$ corresponding to $f \approx 0.037$ (c) as well as $a=2.8$ corresponding to $f \approx 0.086(\mathrm{~d})$, using three wavelets with $l=8, m=3$.

Signal 2 is a multicomponent AM/FM/OM signal given by $g_{2}(\boldsymbol{x})=g_{21}(\boldsymbol{x})+g_{22}(\boldsymbol{x})+\sigma_{2} \epsilon \boldsymbol{x}$, where

$$
\begin{aligned}
g_{21}(\boldsymbol{x}) & \left.=1.2 I\left(x_{1}<N_{1} / 2\right) \cos \left(2 \pi \times 0.087\left(t_{1}^{2} / 2 N_{1}\right)+t_{1}\right)\right) \\
t_{l} & =x_{1} \cos \left(\eta_{l}\right)+\left(x_{1}+x_{2}-155\right) /\left(10 N_{1}\right) \\
\eta_{1} & =-(1 / 4)+\left(x_{1}+x_{2}-155\right) /\left(10 N_{1}\right) \\
g_{22}(\boldsymbol{x}) & =0.8 \cos \left(0.05 \pi\left(t_{2}^{2} /\left(10 N_{1}\right)+t_{2}\right)\right) \\
\eta_{2} & =(\pi / 5) \\
\sigma_{2} & =0.3 \\
N_{1}=N_{2} & =128 .
\end{aligned}
$$

We consider estimating the local orientation at a scale where the more rapid sinusoid is present near the left-hand side (LHS) of the image, and find that the orientation estimate is substantially less noisy when using three wavelets, as is confirmed by Fig. 3 (c) and (d). Clearly, using the multiple wavelets is in both cases substantively decreasing the variability of the estimator.

Finally, let us mention some potential applications: $\mathrm{AM} / \mathrm{FM} / \mathrm{OM}$ signals are often used to model texture [27]. An important problem in this area is segmentation on the basis of the local structure of the signal. The better localization an analysis filter achieves, the more accurately can local texture be characterized. Estimates with reduced variance improves the estimation procedure. We analyze the combination of corduroy textures previously mentioned: see Fig. 4(b). To extract local properties of the image by projecting the image to different scales we find that we may estimate the local orientation of the two different corduroy patterns, see Fig. 4(c) and (d). The low scales Fig. 4(c) extract the vertical oscillations, while the WT at the higher scale, Fig. 4(d) gives the diagonal oscillation.
The examples combine to demonstrate that the Morse wavelets describe discontinuities well, give reduced variance estimates of orientation of oscillatory signals, and the final corduroy pattern illustrated that the good localization of the wavelets allow us to identify different behavior local to different scales. More complicated algorithms, based on reasoning as in [27] and [29] will be investigated in future work.

\section{CONCLUSION}

We have constructed a 2-D localization operator for radial functions and determined its eigensystem. We denoted the eigenfunctions of this operator as isotropic Morse wavelets given they form a natural 2-D extension to the 1-D even Morse wavelets. These 2-D wavelets are optimally localized to the radial Morse region, and we have calculated exact expressions for their localization. Furthermore, in analogy to the 1-D analytic Morse wavelet, we have defined the 2-D monogenic Morse wavelet through the Riesz transform of the isotropic Morse wavelet. The monogenic Morse wavelets constitute a natural framework for determining local directional phase and orientation properties. The monogenic Morse wavelets form a set of orthogonal functions. We have used the orthogonality to establish the statistical properties of the wavelet coefficients of deterministic images immersed in Gaussian white noise. We also discussed the analysis of images formed from aggregations of typical features such as discontinuities and local oscillations. When we average coefficients across wavelets, estimators of local amplitude, phase and orientation of the deterministic image achieve reduced variabilities. Finally, using some examples, we have shown that the theoretical properties of the monogenic Morse wavelets which we derived in this paper hold for discrete implementations.

\section{APPENDIX}

Define the discrete Fourier transform of the noise $\epsilon_{x_{1}, x_{2}}$, via $\mathcal{E}\left(f_{1}, f_{2}\right)$. As the wavelet transform at any angle $\theta$ can be formed from linear combinations of the wavelet transform at $\theta=0$, cf. Section IV-C, only the joint statistical properties of $w_{n_{1}}^{(s)}(\xi ; \epsilon)$; $s=e, 1,2$, need to be found at $\theta=0$. We have

$$
\begin{array}{r}
E\left(w_{n_{1}}^{(e)}(\boldsymbol{\xi} ; \epsilon) w_{n_{2}}^{(e) *}(\boldsymbol{\xi} ; \epsilon)\right) \approx a^{2} \sigma_{1}^{2} \sigma_{2}^{2} \int_{-\left(1 / 2 \Delta_{1}\right)}^{\left(1 / 2 \Delta_{1}\right)} \int_{-\left(1 / 2 \Delta_{2}\right)}^{\left(1 / 2 \Delta_{2}\right)} d^{2} \boldsymbol{f} \\
\times \Psi_{n_{1}}\left(a \sqrt{f_{1}^{2}+f_{2}^{2}}\right) \Psi_{n_{2}}\left(a \sqrt{f_{1}^{2}+f_{2}^{2}}\right)
\end{array}
$$

thus, $E\left(w_{n_{1}}^{(e)}(\boldsymbol{\xi} ; \epsilon) w_{n_{2}}^{(e) *}(\boldsymbol{\xi} ; \epsilon)\right)=\sigma_{\epsilon}^{2} \delta_{n_{1}, n_{2}}$ by (34). Similarly

$$
\begin{aligned}
& \operatorname{Cov}\left(w_{n_{1}}^{(1)}(\boldsymbol{\xi} ; \epsilon), w_{n_{2}}^{(1)}(\boldsymbol{\xi} ; \epsilon)\right) \\
& \approx a^{2} \sigma_{\epsilon}^{2} \int_{-\left(1 / 2 \Delta_{1}\right)}^{\left(1 / 2 \Delta_{1}\right)} \int_{-\left(1 / 2 \Delta_{2}\right)}^{\left(1 / 2 \Delta_{2}\right)} \\
& \quad \times \Psi_{n_{1}}\left(a \sqrt{f_{1}^{2}+f_{2}^{2}}\right) \Psi_{n_{2}}\left(a \sqrt{f_{1}^{2}+f_{2}^{2}}\right) \frac{f_{1}^{2} d f_{1} d f_{2}}{f_{1}^{2}+f_{2}^{2}} \\
& =\frac{1}{2} \sigma_{\epsilon}^{2} \delta_{n_{1}, n_{2}}=\operatorname{Cov}\left(w_{n_{1}}^{(2)}(\boldsymbol{\xi} ; \epsilon), w_{n_{2}}^{(2)}(\boldsymbol{\xi} ; \epsilon)\right) \\
& \operatorname{Cov}\left(w_{n_{1}}^{(e)}(\boldsymbol{\xi} ; \epsilon), w_{n_{2}}^{(1)}(\boldsymbol{\xi} ; \epsilon)\right)
\end{aligned}
$$




$$
\begin{aligned}
& =E\left(w_{n_{1}}^{(e)}\left(a, 0, b_{1} \Delta_{1}, b_{2} \Delta_{2} ; \epsilon\right) w_{n_{2}}^{(1) *}\left(a, 0, b_{1} \Delta_{1}, b_{2} \Delta_{2} ; \epsilon\right)\right) \\
& \approx 0 \\
& =\operatorname{Cov}\left(w_{n_{1}}^{(e)}(\boldsymbol{\xi} ; \epsilon), w_{n_{2}}^{(2)}(\boldsymbol{\xi} ; \epsilon)\right) \\
& =\operatorname{Cov}\left(w_{n_{1}}^{(1)}(\boldsymbol{\xi} ; \epsilon), w_{n_{2}}^{(2)}(\boldsymbol{\xi} ; \epsilon)\right)
\end{aligned}
$$

following from (34). This completes the covariance calculations for the distribution.

\section{ACKNOWLEDGMENT}

The authors would like to express their thanks to the anonymous referees for the many helpful suggestions that substantially improved the paper, as well as their understanding of the topic. They would like to thank Dr. F. Simons for valuable discussions. S. C. Olhede would like to thank Prof. A. Walden for introducing her to this research area.

\section{REFERENCES}

[1] D. Thomson, "Spectrum estimation and harmonic analysis," Proc. IEEE, vol. 70, pp. 1055-96, 1982.

[2] I. Daubechies and T. Paul, "Time-frequency localisation operators: A geometric phase space approach II. The use of dilations and translations," Inverse Problems, vol. 4, pp. 661-80, 1988.

[3] S. C. Olhede and A. T. Walden, "Generalized Morse wavelets," IEEE Trans. Signal Process., vol. 50, pp. 2661-70, 2002.

[4] J. M. Lilly and J. Park, "Multiwavelet spectral and polarization analyses of seismic records," Geophys. J. Int., vol. 122, pp. 1001-21, 1995.

[5] I. Daubechies, Ten Lectures on Wavelets. Philadelphia, PA: Soc. Indust. Appl. Math., 1992.

[6] J.-P. Antoine, R. Murenzi, and P. Vandergheynst, "Directional wavelets revisited: Cauchy wavelets and symmetry detection in patterns," Appl. Computat. Harmon. Anal., vol. 6, pp. 314-45, 1999.

[7] J.-P. Antoine, R. Murenzi, P. Vandergheynst, and S. T. Ali, 2-D Wavelets and Their Relatives. Cambridge, U.K.: Cambridge Univ. Press, 2004.

[8] S. C. Olhede and A. T. Walden, "The Hilbert spectrum via wavelet projections," Proc. R. Soc. Lond. A., vol. 460, pp. 955-75, 2004.

[9] M. Felsberg and G. Sommer, "The monogenic signal," IEEE Trans. Signal Process., vol. 49, pp. 3136-3144, 2001.

[10] B. Boashah, "Estimating and interpreting the instantaneous frequency of a signal-Part; part I: Fundamentals, II: Algorithms," Proc. IEEE, vol. 80, pp. 519-569, 1992.

[11] C. A. Deavours, "The quaternion calculus," The Amer. Math. Month., vol. 80, pp. 995-1008, 1973.

[12] M. Holschneider, Wavelet: An Analysis Tool. Oxford, U.K.: Oxford Science, 1998.

[13] A. V. Oppenheim and J. S. Lim, "The importance of phase in signals," Proc. IEEE, vol. 69, pp. 529-541, 1981.

[14] P. Kovesi, "Invariant measures of image features from phase information," Ph.D., Univ. Western Australia, Perth, Australia, 1996.

[15] W. L. Chan, H. Choi, and R. Baraniuk, "Directional hypercomplex wavelets for multidimensional signal analysis and processing," in IEEE Int. Conf. Acoust. Speech Signal Process. (ICASSP), Houston, TX, May 2004.

[16] F. C. A. Fernandes, R. L. C. van paendonck, and C. S. Burrus, "Multidimensional, mapping-based complex wavelet transforms," IEEE Trans. Image Process., vol. 14, pp. 110-24, 2005.

[17] S. L. Hahn and K. M. Snopek, "Wigner distributions and ambiguity functions of 2-D quaternionic and monogenic signals," IEEE Trans. Signal Process., vol. 53, pp. 3111-28, 2005.
[18] W. Martin and P. Flandrin, "Wigner-ville spectral analysis of nonstationary processes," IEEE Trans. Acoust., Speech Signal Process., vol. 33, pp. 1461-70, 1985.

[19] D. Van de Ville, W. Philips, and I. Lemahieu, "On the N-dimensional extension of the discrete prolate spheroidal window," IEEE Signal Process. Lett., vol. 9, pp. 89-91, 2002.

[20] F. J. Simons, R. D. van der Hilst RD, and M. T. Zuber, "Spatiospectral localization of isostatic coherence anisotropy in Australia and its relation to seismic anisotropy: Implications for lithospheric deformation," J. Geophys. Res., vol. 108 (B5): art, no. 2250, 2003.

[21] F. Hlawatsch, A. Papandreou-Suppappola, and G. F. Bodreaux-Bartels, "The power classes-quadratic time-frequency representations with scale covariance and dispersive time-shift covariance," IEEE Trans. Signal Process., vol. 47, pp. 3067-83, 1999.

[22] S. Dahlke and P. Maass, "The affine uncertainty principle in one and two dimensions," Comp. Math. Applicat., vol. 30, pp. 293-305, 1995.

[23] M. Abramowitz and I. A. Stegun, Eds., Handbook of Mathematical Functions, 9th printing. New York: Dover, 1972.

[24] N. W. McLachlan, Bessel Functions for Engineers. Oxford, U.K.: Clarendon, 1955

[25] S. L. Hahn, "Multidimensional complex signals with single-orthant spectra," Proc. IEEE, vol. 80, pp. 1287-1300, 1992.

[26] T. Bülow and G. Sommer, "Hypercomplex signals-A novel extension of the analytic signal to the multidimensional case," IEEE Trans. Signal Process., vol. 49, pp. 2844-2852, 2001.

[27] A. C. Bovik, N. Gopal, T. Emmoth, and A. Restrepo, "Localized measurement of emergent image frequencies by gabor wavelets," IEEE Trans. Signal Process., vol. 38, pp. 691-712, 1992.

[28] M. Felsberg and G. Sommer, "Structure Multivector for local analysis of images," Christian Albrechts Univ., Kiel, 2001, Tech. Rep. Bericht No 2001.

[29] C. Gonnet and B. Torresani, "Local frequency analysis with 2-D wavelet transform," Signal Process., vol. 37, pp. 389-404, 1994.

[30] S. Lazebnik, C. Schmid, and J. Ponce, A sparse texture representation using local affine regions Beckman Inst., Univ. Illinois at Urbana-Champaign, 2004 [Online]. Available: http://www-cvr.ai. uiuc.edu/ponce_grp/data/texture_database/samples/

[31] G. Metikas and S. C. Olhede, "Multiple wavelet analysis of amplitude/ frequency modulated images," in IEEE Int. Symp. Signal Process. Inf. Technol., Athens, Greece, Dec. 2005.

[32] S. C. Olhede and G. Metikas, "Multiple wavelet coherence analysis," in Proc. IS \& T/SPIE 18th Ann. Symp. 2006, Image Process.: Algorithms Syst., San Jose, CA, Jan. 2006.

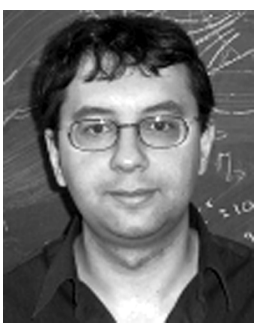

Georgios Metikas received the Ptychion and D.Phil. degrees in theoretical physics from Aristotle University of Thessaloniki, Greece, and Oxford University, U.K., in 1996 and 2000, respectively.

His research interests are theoretical physics and image processing.

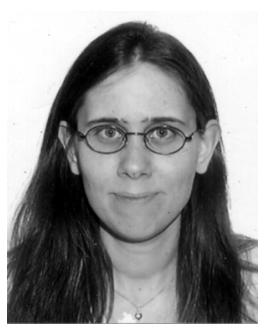

Sofia C. Olhede received the M.Sci. and Ph.D. degrees in mathematics from Imperial College London, U.K., in August 2000 and January 2003, respectively. She joined the Department of Mathematics at Imperial College London in October 2002. Her research interests are nonstationary time series and random field analysis. 